\title{
Structural basis for receptor selectivity and inverse agonism in $\mathrm{S}_{1} \mathrm{P}_{5}$ receptors
}

Elizaveta Lyapina*1, Egor Marin*1,2, Anastasiia Gusach*1,3, Philipp Orekhov ${ }^{1,4,5}$, Andrey Gerasimov ${ }^{6}$, Aleksandra Luginina $^{1}$, Daniil Vakhrameev ${ }^{1}$, Margarita Ergasheva ${ }^{1}$, Margarita Kovaleva ${ }^{1}$, Georgii Khusainov $^{1,7}$, Polina Khorn ${ }^{1}$, Mikhail Shevtsov ${ }^{1}$, Kirill Kovalev ${ }^{1,8}$, Ivan Okhrimenko ${ }^{1}$, Petr Popov ${ }^{1,9}$, Hao $\mathrm{Hu}^{10}$, Uwe Weierstall ${ }^{10}$, Wei Liu ${ }^{11}$, Yunje Cho ${ }^{12}$, Ivan Gushchin ${ }^{1}$, Andrey Rogachev ${ }^{1,13}$, Gleb Bourenkov ${ }^{8}$, Sehan Park ${ }^{14}$, Gisu Park ${ }^{14}$, Hyo Jung Hyun ${ }^{14}$, Jaehyun Park ${ }^{14,15}$, Valentin Gordeliy ${ }^{1,16,17,18}$, Valentin Borshchevskiy ${ }^{1,16,17 \#, \text { Alexey Mishin }}{ }^{1 \#}$, Vadim Cherezov ${ }^{1,19 \# .}$

${ }^{1}$ Research Center for Molecular Mechanisms of Aging and Age-related Diseases, Moscow Institute of Physics and Technology, Dolgoprudny 141701, Russia

${ }^{2}$ Groningen Biomolecular Sciences and Biotechnology Institute, University of Groningen, Nijenborgh 4, 9747 AG Groningen, The Netherlands

${ }^{3}$ Present address: MRC Laboratory of Molecular Biology, Cambridge, CB2 0QH, UK

${ }^{4}$ Faculty of Biology, Lomonosov Moscow State University, Moscow 119991, Russia

${ }^{5}$ Faculty of Biology, Shenzhen MSU-BIT University, Shenzhen 518172, China

${ }^{6}$ Vyatka State University, Kirov 610020, Russia

${ }^{7}$ Present address: Division of Biology and Chemistry, Paul Scherrer Institute, Forschungsstrasse 111, 5232 Villigen PSI, Switzerland

${ }^{8}$ European Molecular Biology Laboratory, Hamburg unit c/o DESY, Hamburg, Germany

${ }^{9}$ Present address: iMolecule, Skolkovo Institute of Science and Technology, Bolshoy Boulevard 30, bld. 1, Moscow 121205, Russia

${ }^{10}$ Department of Physics, Arizona State University, Tempe, AZ 85281, USA

${ }^{11}$ Cancer Center and Department of Pharmacology and Toxicology, Medical College of Wisconsin, Milwaukee, WI 53226, USA

${ }^{12}$ Department of Life Science, Pohang University of Science and Technology, Pohang, Republic of Korea ${ }^{13}$ Joint Institute for Nuclear Research, Dubna 141980, Russia

${ }^{14}$ Pohang Accelerator Laboratory, POSTECH, Pohang 37673, Republic of Korea

${ }^{15}$ Department of Chemical Engineering, POSTECH, Pohang 37673, Republic of Korea

${ }^{16}$ Institute of Biological Information Processing (IBI-7: Structural Biochemistry), Forschungszentrum Jülich, Jülich 52428, Germany

${ }^{17}$ JuStruct: Jülich Center for Structural Biology, Forschungszentrum Jülich, Jülich 52428, Germany

${ }^{18}$ Institut de Biologie Structurale (IBS), Université Grenoble Alpes, CEA, CNRS, Grenoble 38400, France

${ }^{19}$ Bridge Institute, Department of Chemistry, University of Southern California, Los Angeles, CA 90089, USA

*These authors contributed equally: Elizaveta Lyapina, Anastasiia Gusach, Egor Marin.

\#Correspondence and request for materials should be addressed to V.C. (email: cherezov@usc.edu), A.M. (email: mishinalexey@phystech.edu) or V.B. (email: borshchevskiy.vi@phystech.edu). 


\begin{abstract}
The bioactive lysophospholipid sphingosine-1-phosphate (S1P) acts via five different subtypes of S1P receptors $(\mathrm{S} 1 \mathrm{PR})-\mathrm{S}_{1} \mathrm{P}_{1-5} . \mathrm{S}_{\mathrm{P}} \mathrm{P}_{5}$ is predominantly expressed in nervous and immune systems, regulating the egress of natural killer cells from lymph nodes and playing a role in immune and neurodegenerative disorders, as well as carcinogenesis. Several S1PR therapeutic drugs have been developed to treat these diseases; however, they lack receptor subtype selectivity, which leads to side effects. In this article, we describe a $2.2 \AA$ resolution room temperature crystal structure of the human $\mathrm{S}_{1} \mathrm{P}_{5}$ receptor in complex with a selective inverse agonist determined by serial femtosecond crystallography (SFX) at the Pohang Accelerator Laboratory X-Ray Free Electron Laser (PAL-XFEL) and analyze its structure-activity relationship data. The structure demonstrates a unique ligand-binding mode, involving an allosteric subpocket, which clarifies the receptor subtype selectivity and provides a template for structure-based drug design. Together with previously published S1PR structures in complex with antagonists and agonists, the

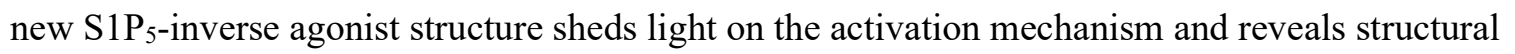
determinants of the inverse agonism in the S1PR-family.
\end{abstract}




\section{INTRODUCTION}

Sphingosine-1-phosphate (S1P) is a lysosphingolipid bio-regulator produced from ceramide in activated platelets, injured cells, and cells stimulated by protein growth factors ${ }^{1,2}$. S1P is released in the blood ${ }^{3}$, where it regulates angiogenesis ${ }^{4}$, cell proliferation, migration, and mitosis ${ }^{5}$ by activating five subtypes of the $\mathrm{S} 1 \mathrm{P}$ G protein-coupled receptors $-\mathrm{S}_{1} \mathrm{P}_{1-5} . \mathrm{S} 1 \mathrm{P}_{1}$ couples only to $\mathrm{G}_{\mathrm{i}}$ protein, $\mathrm{S}_{\mathrm{P}} \mathrm{P}_{4}$ and $\mathrm{S}_{1} \mathrm{P}_{5}$ signal through $\mathrm{G}_{\mathrm{i}}$ and $\mathrm{G}_{12 / 13}{ }^{6}$, and both $\mathrm{S} 1 \mathrm{P}_{2}$ and $\mathrm{S} 1 \mathrm{P}_{3}$ couple to $\mathrm{G}_{\mathrm{i}}, \mathrm{G}_{12 / 13}$, and $\mathrm{G}_{\mathrm{q}}{ }^{7}$. S1P receptors have different expression profiles $-\mathrm{S}_{1} \mathrm{P}_{1}-\mathrm{S}_{1} \mathrm{P}_{3}$ are expressed in all organs throughout the body, while $\mathrm{S}_{1} \mathrm{P}_{4}$ expression is limited to the immune system, and $\mathrm{S} \mathrm{P}_{5}$ is predominantly expressed in the nervous (oligodendrocytes) and immune (NK cells) systems ${ }^{8} . \mathrm{S} \mathrm{P}_{5}$ also inhibits PAR-1 mediated platelet activation ${ }^{9}$. This receptor plays an important role in autoimmune ${ }^{10}$ and neurodegenerative disorders ${ }^{10,11}$ as well as carcinogenesis ${ }^{12}$. For example, $\mathrm{S} 1 \mathrm{P}_{5}$ agonists elicit neuroprotective effects in Alzheimer's and Huntington's diseases ${ }^{10}$, while $\mathrm{S}_{1} \mathrm{P}_{5}$ inhibition leads to apoptosis of cancerous NK cells in large granular leukemia (LGL) ${ }^{12}$. Nonselective modulators such as fingolimod ${ }^{13}$ as well as dual $\mathrm{S}_{1} \mathrm{P}_{1} / \mathrm{S}_{1} \mathrm{P}_{5}$ ligands siponimod ${ }^{14}$ and ozanimod ${ }^{15}$ have been approved for the treatment of multiple sclerosis ${ }^{16}$, Crohn's disease $^{17}$, and other autoimmune

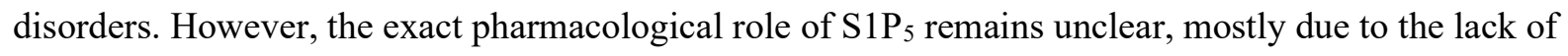
well-characterized potent and highly selective $\mathrm{S}_{1} \mathrm{P}_{5}$ ligands with in vivo activity. While inhibition of $\mathrm{S}_{1} \mathrm{P}_{5}$ is considered as a prospective treatment for $\mathrm{LGL}^{12}$, antagonism of $\mathrm{S}_{1} \mathrm{P}_{1}$ leads to serious adverse effects such as lung capillary leakage, renal reperfusion injury, and cancer angiogenesis ${ }^{18}$. Therefore, highresolution structures of $\mathrm{S} \mathrm{P}_{5}$ in complex with highly selective ligands would shed light on receptor selectivity and provide templates for structure-based design of selective therapeutic drugs with more focused function and fewer side effects.

The first crystal structure of an S1P receptor was published in $2012^{19}$, revealing the inactive state conformation of the human $\mathrm{S}_{1} \mathrm{P}_{1}$ in complex with a selective antagonist sphingolipid mimetic ML056.

This structure helped to select the most promising candidate for clinical trials ${ }^{20}$, which recently culminated in the FDA approval of ozanimod (Zeposia) for the treatment of relapsing multiple sclerosis 
and ulcerative colitis. Recently, a crystal structure of $\mathrm{S}_{1} \mathrm{P}_{3}$ bound to its endogenous agonist ${ }^{21}$ as well as cryo-EM structures of $\mathrm{S}_{1} \mathrm{P}_{1}, \mathrm{~S}_{\mathrm{P}} \mathrm{P}_{3}$, and $\mathrm{S}_{\mathrm{P}} \mathrm{P}_{5}$ in complex with $\mathrm{G}_{\mathrm{i}}$ proteins ${ }^{22,23}$, and $\mathrm{S}_{1} \mathrm{P}_{1}$ in complex with $\mathrm{G}_{\mathrm{i}}$ and $\beta$-arrestin ${ }^{24}$ provided insights in the activation mechanism for the S1P receptor family. However, no structures of this family members in complex with inverse agonists have been reported to date.

In this article, we present the crystal structure of $\mathrm{S}_{\mathrm{P}} \mathrm{P}_{5}$ in complex with a selective inverse agonist ONO$5430608^{25}$ determined by serial femtosecond crystallography (SFX) and analyze it alongside structureactivity relationship data from cell-based functional assays using extensive mutagenesis, molecular docking, molecular dynamics and AlphaFold simulations.

\section{RESULTS}

\section{Structure determination using an X-ray free-electron laser (XFEL)}

Human ${\mathrm{S} 1 \mathrm{P}_{5}}_{5}$ receptor was engineered for crystallization by fusing a thermostabilized apocytochrome b562RIL ${ }^{26}$ into the third intracellular loop (ICL3) and adding a haemagglutinin signal peptide, FLAG tag, and a linker on the $\mathrm{N}$ terminus as well as a PreScission Protease site and decahistidine tag on the $\mathrm{C}$ terminus (Supplementary Fig. 1). Crystals of ${\mathrm{S} 1 \mathrm{P}_{5}}_{5}$ bound to an inverse agonist ONO-5430608 were obtained by lipidic cubic phase (LCP) crystallization ${ }^{27}$ reaching a maximum size of $30 \mu \mathrm{m}$. Attempts to solve the structure were first made using cryocooled crystals at a synchrotron source achieving a maximum of $4 \AA$ single-crystal data resolution, however, the obtained data could not be phased using

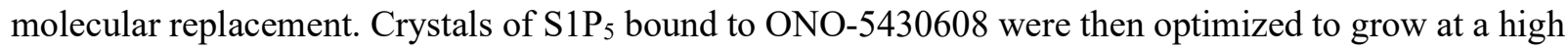
crystal density with an average size of $\sim 5-10 \mu \mathrm{m}$ and used for room temperature SFX data collection at PAL-XFEL (Supplementary Fig. 2). The crystal structure was solved at a $2.2 \AA$ resolution in the P $2{ }_{1} 2_{1} 2_{1}$ space group with identical unit cell constants as the single-crystal data (Supplementary Table 1). A high systematic background scattering from the direct XFEL beam (Supplementary Fig. 3) combined with pseudotranslation led to high structure refinement R-factors, although it did not affect the excellent quality of electron density maps (see Methods and Supplementary Fig. 4). The receptor crystallized with 
two monomers per asymmetric unit, forming an antiparallel dimer through the TM4-TM4 interface (Supplementary Fig. 5).

\section{Inactive conformation of $\mathrm{S}_{5}$ in complex with $\mathrm{ONO}-5430608$}

\section{$\underline{\text { Overall architecture }}$}

The ${\mathrm{S} 1 \mathrm{P}_{5}}_{5}$ structure in complex with ONO-5430608 shares the classical architecture with other class A $\alpha$ branch lipid receptors ${ }^{19,21,28}$, including a heptahelical transmembrane bundle (7TM), two pairs of disulfide bonds stabilizing extracellular loops 2 and 3 (ECL2 and ECL3), an amphipathic C-terminal helix 8 running parallel to the membrane on the intracellular side, and an $\mathrm{N}$-terminal helix capping the ligandbinding site. As expected, the receptor is captured in the inactive conformation (Fig. 1a,b) based on its overall alignment with the inactive state $\mathrm{S}_{1} \mathrm{P}_{1}$ (PDB ID $3 \mathrm{~V} 2 \mathrm{Y}, \mathrm{C} \alpha \mathrm{RMSD}=0.84 / 0.78 \AA$ on $90 \%$ of residues for chains $\mathrm{A} / \mathrm{B}$ of our $\mathrm{S}_{1} \mathrm{P}_{5}$ structure) and the active state $\mathrm{S}_{1} \mathrm{P}_{5}(\mathrm{PDB}$ ID $7 \mathrm{EW} 1, \mathrm{C} \alpha \mathrm{RMSD}=$ $1.40 / 1.40 \AA$ on $90 \%$ of residues for chains $\mathrm{A} / \mathrm{B}$ of our $\mathrm{S}_{1} \mathrm{P}_{5}$ structure) as well as on the conformation of conserved activation-related motifs described in the following section.

\section{Activation-related conserved motifs and Na-binding site}

A dual toggle switch $\mathrm{L}(\mathrm{F})^{3.36}-\mathrm{W}^{6.48}$ (superscripts refer to the Ballesteros-Weinstein ${ }^{29}$ residue numbering scheme in class A GPCRs) together with $\mathrm{P}^{5.50}-\mathrm{I}^{3.40}-\mathrm{F}^{6.44}$ motif have been characterized as the common microswitches in class A GPCRs that transmit activation-related conformational changes from the ligandbinding pocket towards an outward movement of TMs 5 and 6 and inward displacement of TM7 on the intracellular side ${ }^{30,31}$. In all S1P receptors, the dual toggle switch is conserved as $\mathrm{L}^{3.36}-\mathrm{W}^{6.48}$; however, the first two residues of the P-I-F motif deviate from the consensus (Fig. 1e). Nevertheless, the I.50-V $\mathrm{V}^{3.40}-\mathrm{F}^{6.44}$ motif in $\mathrm{S}_{1} \mathrm{P}_{5}$ appears to serve a similar role as the classical P-I-F motif in other receptors, as the sidechains of $\mathrm{V}^{3.40}$ and $\mathrm{F}^{6.44}$ switch over upon activation. The I-V-F switch in $\mathrm{S}_{1} \mathrm{P}_{5}$ is connected to the dual toggle switch through steric interactions between $\mathrm{F}^{6.44}$ and $\mathrm{W}^{6.48}$, and the shift of $\mathrm{W} 264^{6.48}$ is accompanied by a rotamer switch of $\mathrm{L} 119^{3.36}$ (Fig. 1c). Similar dual (also known as "twin") toggle switch 
$\mathrm{L}(\mathrm{F})^{3.36}-\mathrm{W}^{6.48}$ has been shown to play a key role in the activation of several other receptors, such as $\mathrm{CB} 1^{32,33}, \mathrm{AT}^{34}$, and $\mathrm{MC}^{35}$.

An allosteric sodium-binding site located in the middle of the 7TM bundle near $\mathrm{D}^{2.50}$ is highly conserved in class A GPCRs ${ }^{36}$. Binding of a $\mathrm{Na}^{+}$ion along with several water molecules in this site stabilizes the inactive receptor conformation. Upon receptor activation, the pocket collapses, likely expelling $\mathrm{Na}^{+}$into the cytoplasm ${ }^{36,37}$. Despite a relatively high resolution and conservation of critical sodium binding residues, such as $\mathrm{D} 82^{2.50}, \mathrm{~S} 122^{3.39}$, and $\mathrm{N} 298^{7.45}$, we could not locate a $\mathrm{Na}^{+}$in the electron density of $\mathrm{S} \mathrm{P}_{5}$, most likely because of a low sodium concentration in the final crystallization buffer ( $20 \mathrm{mM})$. Other residues lining the $\mathrm{Na}^{+}$-binding pocket $\left(\mathrm{N}^{1.50}, \mathrm{~S}^{3.39}, \mathrm{~N}^{7.45}, \mathrm{~S}^{7.46}, \mathrm{~N}^{7.49}, \mathrm{Y}^{7.53}\right)$ are also conserved in $\mathrm{S}_{1} \mathrm{P}_{5}$, with the exception of two polar residues, $\mathrm{T} 79^{2.47}$ and $\mathrm{S} 81^{2.49}$, in the side part of the pocket, which are typically represented by two hydrophobic alanines ${ }^{36}$.

On the intracellular side of the receptor, conserved residues E132 $32^{3.49}$ and R133 $3^{3.50}$ of the D[E]RY motif form an ionic lock that stabilizes the inactive state (Fig. 1d). Upon receptor activation, this ionic lock breaks apart releasing R133 $3^{3.50}$ for interaction with a $\mathrm{G}$ protein ${ }^{38,39}$. Interestingly, $\mathrm{S}_{1} \mathrm{P}_{5}$ possesses $\mathrm{S}_{134} 4^{3.51}$ in this motif, which is seen in only 6 class A receptors out of 714, compared to a more common residue $\mathrm{Y}$ that is present in $66 \%$ of class A receptors.

\section{Ligand-binding pocket}

\section{Overall structure of the ligand-binding pocket}

The co-crystallized ligand ONO-5430608 (4-\{6-[2-(1-naphthyl)ethoxy]-1,2,4,5-tetrahydro-3H-3benzazepin-3-yl \} butanoic acid) has been developed within a series of $\mathrm{S} \mathrm{P}_{5}$-selective modulators ${ }^{25}$ and characterized as a potent inverse agonist at $\mathrm{S}_{1} \mathrm{P}_{5}$ in $\mathrm{G}_{\mathrm{i}}$ protein mediated cAMP accumulation assay $\left(\mathrm{EC}_{50}\right.$ $=1.7 \mathrm{nM})$ (Supplementary Fig. 6 and Table 2). The ligand was modeled in a strong electron density observed inside the ligand-binding pockets of both receptor molecules in the obtained crystal structure (Fig. 2a and Supplementary Fig. 4). The overall architecture of the pocket, shared by other members of the S1PR family, reflects both zwitterionic and amphipathic properties of the endogenous S1P ligand ${ }^{19,21}$. 
The pocket is occluded on the extracellular side by the N-terminal alpha-helix packed along ECL1 and ECL2, with a small opening between TM1 and TM7 (Fig. 1f), which has been proposed to serve as the entrance gate for lipid-like ligands ${ }^{19}$. The orthosteric ligand-binding pocket in $\mathrm{S}_{1} \mathrm{P}_{5}$ consists of a polar charged part, composed of residues from the N-terminal helix and extracellular tips of TM2 and TM3 that interact with the zwitterionic headgroup of S1P, as well as a hydrophobic cavity, lined up by hydrophobic and aromatic residues, which accommodates the alkyl tail of S1P (Fig. 2b). The negatively charged butanoic acid group of ONO-5430608 occupies the polar part of the pocket mimicking the phosphate group of S1P, the core tetrahydro-benzazepine rings fill in space in the middle of the pocket, while the naphthyl-ethoxy group unexpectedly swings over and extends into a previously unidentified allosteric sub-pocket. The sub-pocket is surrounded by non-conserved residues from TM1, TM2, and TM7 and opened in our structure due to a rotameric switch of $\mathrm{Y} 89^{2.57}$ compared to structures of other S1P receptors (Fig. 2a,b). The distinct amino acid composition of this allosteric sub-pocket suggests that it can serve as a selectivity determinant for $\mathrm{S}_{1} \mathrm{P}_{5}$-specific ligands and makes the hallmark of the structure described in this work.

\section{Functional characterization of the ligand binding hotspots in $\mathrm{S}_{1} \mathrm{P}_{5}$}

To validate the observed ligand binding pose and further expand our knowledge about the ligand selectivity and relative importance of specific residues, we tested 25 structure-inspired ligand-binding pocket mutants of $\mathrm{S} \mathrm{P}_{5}$ by a BRET-based cAMP production assay using the endogenous agonist $\mathrm{S} 1 \mathrm{P}$ and the co-crystallized inverse agonist ONO-5430608 (Fig. 2c, Supplementary Table 2, and Supplementary Fig. 7). In line with the binding pocket structure description given above, we consequently characterize important interactions in each part.

\section{Polar part of the orthosteric pocket}

The polar upper part of the binding pocket is highly conserved among the whole S1PR family (Fig. 2b). It consists of residues $\mathrm{Y} 19^{\mathrm{N}-\mathrm{Term}}, \mathrm{K} 24^{\mathrm{N}-\mathrm{Term}}, \mathrm{N} 92^{2.60}, \mathrm{R} 111^{3.28}$, and $\mathrm{E} 112^{3.29}$ and accommodates the phosphate and primary amine groups of S1P. The receptor's potential for multiple polar interactions in this region is 
utilized in anchoring zwitterionic groups of synthetic ligands of S1P receptors. Thus, in our $\mathrm{S}_{1} \mathrm{P}_{5}$ structure, the carboxyl group of ONO-5430608 is stabilized by polar interactions with Y19 $9^{\mathrm{N}-\text { term }}, \mathrm{K} 24^{\mathrm{N}-\text { term }}$, and $\mathrm{R} 111^{3.28}$, while the protonated tertiary amine group makes a salt bridge with E1 $12^{3.29}$, similar to

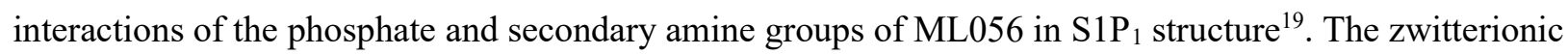
headgroup of the endogenous $\mathrm{S} 1 \mathrm{P}$ ligand bound to $\mathrm{S}_{1} \mathrm{P}_{3}$ is shifted towards $\mathrm{TM} 1$, while retaining the same interactions except for the $\mathrm{N}$-terminal $\mathrm{K} 27^{21}$.

The mutations disrupting polar interactions with zwitterionic ligand head groups: Y19 ${ }^{\mathrm{N}-\mathrm{Term}} \mathrm{A} / \mathrm{F}, \mathrm{K} 24^{\mathrm{N}-\mathrm{Term}}$ $\mathrm{A} / \mathrm{Q}, \mathrm{N} 92^{2.60} \mathrm{~A} / \mathrm{C}, \mathrm{R} 111^{3.28} \mathrm{~A} / \mathrm{Q}$, and $\mathrm{E} 112^{3.29} \mathrm{~A} / \mathrm{Q}$ either fully abolish or significantly (by an order of magnitude or more) decrease the response for both ONO-5340608 and S1P (Fig. 2c and Supplementary Table 2). Notably, some mutations have different effects on S1P and ONO-5430608. While mutations of $\mathrm{N} 92^{2.60}, \mathrm{R} 111^{3.28}$, and $\mathrm{E} 112^{3.29}$ completely eliminate response to the inverse agonist, they only decrease the potency for S1P. A similar effect of mutations of homologous amino acids on S1P potency was previously observed for $\mathrm{S}_{3}{ }_{3}{ }^{21}$. In this case, each of the three amino acids independently interacts with the amine group of S1P (see PDB ID 7C4S). On the other hand, in our S1P ${ }_{5}-\mathrm{ONO}-5430608$ structure, these three amino acids are interconnected and form a stable cluster which further interacts with the tertiary amine and the carboxyl group of ONO-5430608. Thus, mutations of any of the three amino acids

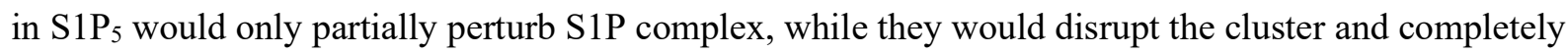
eliminate the binding of ONO-5430608.

Although the locations of residues, known to interact with the phosphate group of S1P from either functional or structural data, are largely conserved between S1P receptors, the effects of their mutations on S1P potency are different. Namely, mutations of N-terminal Y29/19 and K34/24 to alanine render ${\mathrm{S} 1 \mathrm{P}_{1}}_{1} / \mathrm{S}_{1} \mathrm{P}_{5}$, respectively, non-responsive to $\mathrm{S} \mathrm{P}^{19}$, while corresponding mutations preserve the interaction with $\mathrm{S}_{1} \mathrm{P}_{3}{ }^{21}$. These data suggest a different orientation of the phosphate headgroup of S1P within the binding pocket in different receptors.

\section{Hydrophobic part of the orthosteric pocket}


The hydrophobic part of the orthosteric binding pocket in S1P receptors accommodates the lipidic tail of the endogenous ligand or its synthetic analogs such as ML056 $6^{19,21,40}$. The residues on its bottom are conserved among S1PRs (Fig. 2b) and well characterized ${ }^{21,40}$. The top part of the hydrophobic subpocket in $\mathrm{S}_{1} \mathrm{P}_{5}$, which in our structure accommodates the tetrahydro-benzazepine double ring system of ONO5430608 , consists of residues V115 $5^{3.32}$, L292 $2^{7.39}$, and Y $89^{2.57}$ that are less characterized, although they play an important role in ligand binding.

In our functional assays, mutations of $\mathrm{V} 115^{3.32}$ to A and L decrease potencies of both S1P and ONO5430608 (Fig. 2c and Supplementary Table 2). Additionally, Y89 ${ }^{2.57} \mathrm{~A}$ abolishes the functional response of both ligands, while $\mathrm{Y} 89^{2.57} \mathrm{~W}$ preserves it, suggesting that an aromatic residue is crucial at this position.

\section{Allosteric sub-pocket}

Although ONO-543060 shares a similar zwitterionic headgroup with other co-crystallized S1PR ligands, its hydrophobic tail is substantially different. The bulky naphthyl group of ONO-543060 does not fit well in the relatively narrow hydrophobic cleft of the orthosteric pocket and instead accommodates a previously uncharacterized allosteric subpocket between TM1 and TM7 (Fig. 2a,b). The subpocket is formed by non-conserved hydrophobic residues $C 43^{1.39}$ (90 $\AA^{2}$ occluded area), $193^{2.61}\left(127 \AA^{2}\right)$, L292.39 $\left(126 \AA^{2}\right), G 293^{7.40}\left(50 \AA^{2}\right)$, and M296 $6^{7.43}\left(123 \AA^{2}\right)$. Site-directed mutagenesis of residues in the allosteric pocket and functional data suggest a strong role of TM7 residues of the pocket in ligand binding. In particular, mutations L292 $2^{7.39} \mathrm{~A} / \mathrm{V}$ and $\mathrm{M} 296^{7.43} \mathrm{~V} / \mathrm{W}$ decrease ONO-543060 potency by over an order of magnitude (Fig. 2c). On the other hand, mutations $\mathrm{C} 433^{1.39} \mathrm{~F}$ and $\mathrm{G} 293^{7.40} \mathrm{~A}$ show almost no effect on either ligand binding. The strengths of the effects appear to correlate with the occluded areas of residues interacting with ONO-543060, as calculated from the crystal structure.

Meta molecular dynamics simulations of $\mathrm{Y}^{2.57}$ conformational flexibility

The allosteric subpocket displays a large variability in its residues among S1P receptors (Fig. 2b), likely contributing to the exceptional selectivity of ligands targeting it. Interestingly, this pocket is present in our $\mathrm{S} 1 \mathrm{P}_{5}$ structure largely due to the flip of one amino acid, Y89 ${ }^{2.57}$, compared to other S1PR structures. We 
thus explored the conformational flexibility of $\mathrm{Y}^{2.57}$ in the available structures of $\mathrm{S}_{1} \mathrm{P}_{1}, \mathrm{~S}_{1} \mathrm{P}_{3}$, and $\mathrm{S}_{1} \mathrm{P}_{5}$ receptors using an enhanced molecular dynamics simulation technique, originally developed by Laio and Parrinello ${ }^{41}$ and known as metadynamics (metaMD), as well as by targeted mutagenesis.

MetaMD facilitates sampling of the free energy landscape along the selected reaction coordinate(s), e.g., a torsion angle, by adding biasing potentials (most commonly positive Gaussians) driving the system out of local minima. By adding multiple Gaussians, the system is discouraged to return to already sampled regions of the configurational space what eventually allows it to escape free energy minima. The free energy landscape can be then recovered as the opposite of the cumulative biasing potential. Here, we used metaMD to estimate free energy profiles along the reaction coordinate corresponding to the torsion rotation of the $\mathrm{Y}^{2.57}$ side chain.

The free energy profile of the $\mathrm{Y} 89^{2.57}$ side chain torsion in $\mathrm{S} \mathrm{P}_{5}$ features two minima (Fig. 3a,c): the global minimum corresponds to a downward orientation of Y89 $9^{2.57}$ as observed in our crystal structure, while the second minimum at a higher energy level is close to an upward orientation of $\mathrm{Y}^{2.57}$ found in the $\mathrm{S} 1 \mathrm{P}_{1}$ and ${\mathrm{S} 1 \mathrm{P}_{3}}_{3}$ crystal structures. On the other hand, the free energy profile of the $\mathrm{Y}^{2.57}$ side chain torsion in both $\mathrm{S}_{1} \mathrm{P}_{1}$ and $\mathrm{S}_{1} \mathrm{P}_{3}$ has only a single minimum near their crystallographic upward conformations (Fig. 3c). The downward orientation of $\mathrm{Y}^{2.57}$ in the latter case is likely hampered by steric clashes with $\mathrm{M}^{3.32} / \mathrm{V}^{7.43}$, making this conformation energetically unfavorable. $\mathrm{S} \mathrm{P}_{5}$ has a smaller valine in position 3.32, which does not interfere with the downward orientation of $\mathrm{Y}^{2.57}$. At the same time, a more flexible methionine in position 7.43 may swap positions with $Y 89^{2.57}$ allowing the latter to switch between the upward and downward conformations.

\section{Insights from molecular docking}

To further explore structure-activity relationships from both ligand and protein points of view, we performed molecular docking calculations. To assess the importance of the Y89 $9^{2.57}$ conformation in ligand binding, we compared two $\mathrm{S}_{1} \mathrm{P}_{5}$ models: the crystal structure (Y89 $9^{2.57}$ in the downward conformation) and a metaMD snapshot (Y89 2.57 in the upward conformation) in their ability to predict binding of ONO- 
5430608 ligand series ${ }^{25}$. For the crystal structure, the docking scores correlate well with the ligand affinity: the most potent group 'A' ligands ( $\mathrm{IC}_{50}$ between 1 and $100 \mathrm{nM}$ ) have docking scores of $-37 \pm 5$, whereas the least potent group ' $\mathrm{C}$ ' ligands ( $\mathrm{IC}_{50}$ between 1 and $3 \mu \mathrm{m}$ ) have scores $-23 \pm 4$, and for the intermediate group ' $\mathrm{B}$ ' $\left(\mathrm{IC}_{50}\right.$ between $100 \mathrm{nM}$ and $\left.1 \mu \mathrm{M}\right)$ scores are $-32 \pm 5$ (Supplementary Fig. 7b). The docking poses of group 'A' compounds closely resemble the ligand pose in the crystal structure (Supplementary Fig. 7a). Namely, the interactions of the negatively-charged headgroup with Y19/K24, as well as the interaction of the positively-charged amino group with E112 $2^{3.29}$, and the position of the double-ring system are preserved. For the upward conformation of $Y 89^{2.57}$, the docking scores show no correlation with the ligand affinity, and the docking poses of the group "A" ligands show no consistency between each other and the obtained data from the mutation screening (Supplementary Fig. 7c-d).

Notably, the SAR data for the ONO-5430608 ligand series suggest a role of the substituent position on the core double-ring system in the ligand binding. Namely, most of the lower affinity ligands (group "C") have a tetrahydroisoquinoline or tetrahydronaphthalene scaffold instead of the tetrahydro-benzazepine, which is more common among group "A" and "B" ligands. Likely, the affinity drop occurs due to the overall ligand shape, rather than the ring size. Namely, most ligands with both substituents placed on the same side of the middle plane across the double-ring system display higher affinity, while all ligands with two substituents placed on different sides have a low affinity (Supplementary Fig. 8), with the only exception, Example 18-2, which however has an amine group placed within the isoquinoline system, compared to other same-side substituents ligands. This notion also suggests a common framework for designing $\mathrm{S} 1 \mathrm{P}_{5}$-selective ligands.

\section{$\underline{\text { Inverse agonism }}$}

It has been shown that $\mathrm{S}_{1} \mathrm{P}_{5}$ exhibits a relatively high level of basal activity ${ }^{42}$, while our functional assay revealed that ONO-5430608 acts as an inverse agonist for the $\mathrm{G}_{\mathrm{i}}$-protein-mediated signaling pathway, reliably decreasing the basal activity level detected by the BRET-based cAMP sensor (Supplementary Fig. 6). 
Our ${\mathrm{S} 1 \mathrm{P}_{5}}_{5}$ structure in complex with the inverse agonist ONO-5430608 along with previously reported agonist and antagonist-bound structures of $\mathrm{S}_{1} \mathrm{P}_{1,3,5}$ shed light on the mechanism of inverse agonism. Specifically, the above-mentioned conformational flexibility of Y $89^{2.57}$ may provide a structural background for the basal activity of $\mathrm{S} \mathrm{P}_{5}$. As shown by metaMD, the upward orientation of $\mathrm{Y}^{2} 9^{2.57}$ is compatible with both active and inactive conformations of the dual toggle switch $\mathrm{L}^{3.36}-\mathrm{W}^{6.48}$, while the downward orientation of $\mathrm{Y} 89^{2.57}$ selects the inactive conformation (Fig. 3d). The dual toggle switch is found in the active state in the previously reported structures of $\mathrm{S}_{1} \mathrm{P}_{5}$-siponimod as well as in $\mathrm{S}_{1} \mathrm{P}_{1}{ }^{23}$ and $\mathrm{S}_{1} \mathrm{P}_{3}{ }^{21}$ agonist-bound complexes. It induces activation of the P-I-F-motif and an outward movement of the intracellular part of TM6 resulting in G-protein signaling cascade. On the other hand, the dual toggle switch is observed in the inactive conformation in our $\mathrm{S}_{1} \mathrm{P}_{5}-\mathrm{ONO}-5430608$ structure and in the previously published antagonist-bound $\mathrm{S}_{1} \mathrm{P}_{1}{ }^{19}$. The inverse agonist ONO-5430608 induces the downward conformation of $Y 89^{2.57}$ that opens the allosteric subpocket and suppresses the switching of L119.36 locking the dual toggle switch in the inactive state (Fig. 3b). Therefore, conformational flexibility of $\mathrm{Y}^{2} 9^{2.57}$ in $\mathrm{S}_{1} \mathrm{P}_{5}$ provides structural basis for both receptor subtype selectivity and inverse agonism.

\section{Naturally occurring mutations in $\mathrm{S}_{1} \mathrm{P}_{5}$}

In order to characterize additional functionally important residues in $\mathrm{S}_{1} \mathrm{P}_{5}$, we performed mapping of known point mutations from genomic databases onto the crystal structure (Supplementary Fig. 9). Multiple databases carry information about $\mathrm{S} \mathrm{P}_{5}$ point mutations including gnomAD $(229 \mathrm{SNVs})^{43}$, which contains genomic information from unrelated individuals, and COSMIC (124 point mutations) ${ }^{44}$, which accumulates somatic mutations in cancer. The most frequent gnomAD mutation $\mathrm{L} 318^{8.55} \mathrm{Q}$ in helix 8 ( $3 \%$ of the population) was shown to impair $\mathrm{G}_{12}$ signaling ${ }^{45}$; however, according to our functional data it only slightly decreases the potency of $\mathrm{S} 1 \mathrm{P}$ in $\mathrm{G}_{\mathrm{i}}$ mediated signaling (Fig. 2c). It was previously proposed $^{45}$ that a possible cause of this mutation on the signaling impairment is the prevention of palmitoylation of the downstream $\mathrm{C} 322^{8.59}$ or $\mathrm{C} 323^{\mathrm{C}-\text { term }}$. A concomitant cause might be a shift in the helix 8 position due to the loss of a hydrophobic contact between the mutated residue L $318^{8.55}$ and the membrane. 
Several individuals have missense mutations in the ligand binding pocket; for example, 3 out of 235,080 samples $^{43}$ contain $\mathrm{R} 111^{3.28} \mathrm{~L}$ mutation possibly affecting the contacts with the zwitterionic ligand headgroup (Fig. 2). Mutation of another headgroup recognition residue, E1 $12^{3.29} \mathrm{G}$, is less frequent (1 of 234,568). As shown in our functional data, mutations of both of these residues to neutral ones disrupt response to ligands (Fig. 2c). Additionally, two mutations located side-by-side in the putative ligand entrance gateway, $\mathrm{C} 43^{1.39} \mathrm{~F}$ and $\mathrm{M} 296^{7.43} \mathrm{~V}$, are present in the population ${ }^{43}$ at $10^{-6}$ frequencies. While $\mathrm{C} 43^{1.39} \mathrm{~F}$ shows little effect in our functional tests, $\mathrm{M} 296^{7.43} \mathrm{~V}$ disrupts $\mathrm{G}_{\mathrm{i}}$ signaling response for both S1P and ONO-5430608 (Fig. 2c). Another conserved in S1P receptors, except for $\mathrm{S} 1 \mathrm{P}_{2}$, residue $\mathrm{A} 295^{7.42}$ has a hydrophobic contact with the ligand (Fig. 2a), which becomes altered in case of the A295 ${ }^{7.42}$ S mutation. Mutation of A295 $5^{7.42}$ S may also directly influence the state of the toggle switch (L119 $9^{3.36}-\mathrm{W} 264^{6.48}$ in $\left.\mathrm{S}_{1} \mathrm{P}_{5}\right)$ and may interfere with protein activation, as it was shown for several other receptors, e.g., $\beta_{2}-$ adrenergic receptor ${ }^{46}$ and CCR $5^{47}$. One of the key residues in the sodium binding site, $\mathrm{N} 298^{7.45}$, has several variations in population: $\mathrm{S}, \mathrm{D}$, or K. While the effects of $\mathrm{N} 298^{7.45} \mathrm{D}$ and $\mathrm{N} 298^{7.45} \mathrm{~S}$ are unclear, $\mathrm{N} 298^{7.45} \mathrm{~K}$ would mimic sodium binding, stabilizing the inactive state of the receptor ${ }^{48}$.

Somatic mutations appearing in COSMIC and not found in the population may be linked to severe cancer impairments. For example, $\mathrm{S} 125^{3.42} \mathrm{R}$ disrupts the conservative hydrogen-bond network involving S77 2.45 and $\mathrm{W} 159^{4.50}$, destabilizing contacts between TMs 2,3 , and $4^{49}$ and, likely, disturbing the 7TM fold due to the introduction of a charged residue in a mostly hydrophobic environment.

\section{Comparison with AlphaFold predictions}

Recently, a redesigned artificial intelligence-based protein structure-predicting system AlphaFold v. $2^{50}$ achieved an outstanding breakthrough in approaching the accuracy in protein structure modeling previously available only from experimental methods. AlphaFold-based approaches started to find multiple applications in structural biology ${ }^{51}$, however, their full capacity and limitations remain to be uncovered. Here, we evaluated the ability of AlphaFold to predict structural features responsible for receptor selectivity and inverse agonism in the S1PR family. For that, we generated 50 de novo 
AlphaFold models for each of the five S1PRs. Overall, the models demonstrated reasonable correspondence to the available experimental structures; for example, $\mathrm{C} \alpha \mathrm{RMSDs}$ in the 7TM region between the $\mathrm{S}_{1} \mathrm{P}_{5}$ models and the inactive state crystal structure ( $\left.\mathrm{S}_{1} \mathrm{P}_{5}-\mathrm{ONO}-5430608\right)$ is $1.3 \pm 0.2 \AA$ and

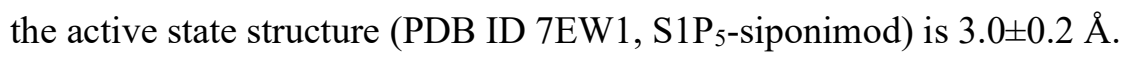

The conformational heterogeneity of $\mathrm{Y}(\mathrm{F})^{2.57}$ observed in experimental S1PR structures and metaMD simulations was also well captured by AlphaFold predictions (Supplementary Fig. 10a-f). In all S1P ${ }_{1}$, $\mathrm{S}_{1} \mathrm{P}_{3}$, and $\mathrm{S}_{1} \mathrm{P}_{4}$ models, $\mathrm{Y}^{2.57}$ has an upward conformation, except for a single $\mathrm{S}_{1} \mathrm{P}_{1}$ model, in which this residue adopts a downward orientation similar to that previously observed in all-atom MD simulations ${ }^{52}$. Furthermore, 19 out of $50 \mathrm{~S} \mathrm{P}_{5}$ models display a downward $\mathrm{Y}^{2.57}$ orientation, while all the others have an upward $\mathrm{Y}^{2.57}$ orientation. Notably, $\mathrm{S}_{1} \mathrm{P}_{2}$ is the only receptor, in which $\mathrm{Y}^{2.57}$ is replaced with $\mathrm{F}^{2.57}$ that adopts a downward conformation in all generated models. The downward orientation of $\mathrm{F}^{2.57}$ in $\mathrm{S}_{1} \mathrm{P}_{2}$, similar to that of $\mathrm{Y}^{2.57}$ in $\mathrm{S}_{1} \mathrm{P}_{5}$, opens the allosteric subpocket, which may be targeted to achieve ligand selectivity.

In all available experimental S1PR structures, the conserved dual toggle switch $\mathrm{L}^{3.36}-\mathrm{W}^{6.48}$ displays either active or inactive conformation. AlphaFold predicted both of these conformations for all receptors except for $\mathrm{S}_{1} \mathrm{P}_{4}$, in which only the active conformation was present in all models (Supplementary Fig. 10a-e). However, AlphaFold models did not fully reflect the mutual relationship between conformations of Y89 $9^{2.57}$ and L119 ${ }^{3.36}$, as observed by metaMD in $\mathrm{S}_{\mathrm{P}}$. Thus, all AlphaFold-predicted $\mathrm{S}_{1} \mathrm{P}_{5}$ models cluster into three groups (Supplementary Fig. 10e), including the energetically unfavorable conformation with Y $89^{2.57}-\mathrm{L} 119^{3.36}$ in downward-upward orientations while missing the energetically favorable conformation with Y89 2.57-L1193.36 in upward-downward positions. Consequently, we conclude that the current version of AlphaFold could not consistently generate an S1PR structure in a specific signaling state, sometimes mixing the features of different conformations in a single model. These findings are corroborated by a recent study of several other GPCRs ${ }^{53}$. 
One of the most intriguing AlphaFold-related questions is how useful are the predicted models for structure-based drug design ${ }^{54}$. To test it in application to S1PR targets, we constructed three benchmarks, mimicking virtual ligand screening campaigns, and compared the available experimental structures and AlphaFold models by their ability to distinguish high-affinity ligands from low-affinity binders and decoys. Our results demonstrated that crystal structures outperform AlphaFold-generated models in several scenarios (Supplementary Fig. 11). Namely, our S1 $\mathrm{P}_{5}$ crystal structure showed substantially better overall ranking and top-10\% enrichment among both ONO-5430608-like inverse agonists (“ONO”

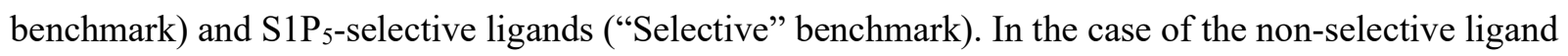
benchmark (mostly S1P 1 agonists), the best performance was achieved for several experimental S1PR structures determined in complex with non-selective ligands, e.g., $\mathrm{S}_{\mathrm{P}} \mathrm{P}_{1}$-siponimod complex (Supplementary Fig. 11), while our $\mathrm{S} 1 \mathrm{P}_{5}$ structure fared on par with AlphaFold models.

\section{DISCUSSION}

Here, we present the $2.2 \AA$ crystal structure of the human $\mathrm{S}_{1} \mathrm{P}_{5}$ receptor in complex with its selective inverse agonist. The structure was obtained by room temperature SFX data collection at PAL-XFEL using sub-10 $\mu \mathrm{m}$ crystals. In combination with site-directed mutagenesis, functional assays, metaMD simulations and docking studies, this structure revealed molecular determinants of ligand binding and selectivity as well as shed light on the mechanism of inverse agonism in the S1PR family. The obtained structure also allowed us to map locations of known missense SNVs from gnomAD and COSMIC genome databases and annotate their potential functional roles providing future insights in personalized medicine approaches.

We found that the inverse agonist ONO-5430608 binds to the receptor's orthosteric site, suppressing $\mathrm{S}_{1} \mathrm{P}_{5}$ basal activity. Highly conserved residues $\mathrm{Y} 19^{\mathrm{N}-\text { term }}, \mathrm{K} 24^{\mathrm{N}-\text { term }}, \mathrm{R}^{3.28}$, and $\mathrm{E}^{3.29}$ play an essential role in the recognition of both ONO-5430608 and its native ligand S1P. The naphthyl group of ONO-5340608 occupies an allosteric subpocket that was not previously observed in any other S1PR structure. While the orthosteric site is highly conserved in the S1PR family, the allosteric subpocket is composed of unique 
residues and is present in our $\mathrm{S}_{1} \mathrm{P}_{5}$ structure due to the conformational switch of a single residue $\mathrm{Y}^{2.57}$. Functionally important residues were revealed by structure-guided site-directed mutagenesis and $\mathrm{G}_{\mathrm{i}}$ signaling assays. We further used metaMD simulations to explore the conformational flexibility of $\mathrm{Y}^{2.57}$ in S1PRs and established its role in receptor subtype selectivity and inverse agonism. The role of $\mathrm{Y}^{2.57}$ in binding of selective ligands was also confirmed by comparative molecular docking simulations. Furthermore, taking advantage of the availability of several experimental structures of S1PRs in different functional states, we tested the ability of AlphaFold to predict de novo specific conformational states for S1PRs and to provide reliable templates for structure-based virtual ligand screening. While the AlphaFold-generated models showed a close similarity to experimental structures and captured conformational diversity of conserved structural motifs, the models did not provide a full description of specific signaling states and showed subpar performance in virtual ligand screening compared to experimental structures.

Our structure along with our functional and computer modeling data may facilitate the rational design of ligands that could further serve as lead or tool compounds for detailed elucidation of biological function of $\mathrm{S}_{1} \mathrm{P}_{5}$ and therapeutic developments. $\mathrm{S}_{1} \mathrm{P}_{5}$ is emerging as a promising drug target. Inhibiting $\mathrm{S}_{1} \mathrm{P}_{5}$ by an inverse agonist could create new therapeutic strategies against neuroinflammation and degeneration where the high ligand selectivity would diminish the off-target effects. While $\mathrm{S}_{1} \mathrm{P}_{1}$ has a broad expression profile, ${\mathrm{S} 1 \mathrm{P}_{5}}_{5}$ is expressed predominantly in brain tissues ${ }^{8}$; thus, a highly selective compound would afford more localized control over associated CNS disorders not affecting peripheral processes in the body. 


\section{METHODS}

\section{Protein engineering for structural studies}

The human wild type gene S1PR5 (UniProt ID Q9H228) was codon-optimized by Genescript for insect cell expression and modified by adding a hemagglutinin signal peptide (HA; KTIIALSYIFCLVFA), a FLAG-tag for expression detection, and an Ala-Gly-Arg-Ala linker at the N-terminus. An apocytochrome b562RIL (BRIL) ${ }^{26}$ was inserted in the third intracellular loop between A223 and R241 to stabilize the receptor and facilitate crystallization. The C-terminus was truncated after Val321, and a PreScission cleavage site was added after it to enable removal of the following 10xHis tag used for IMAC purification (Supplementary Fig. 1). The resulting construct was cloned into a pFastBac1 (Invitrogen) plasmid. The full DNA sequence of the $\mathrm{S}_{1} \mathrm{P}_{5}$ crystallization construct is provided in Supplementary Table 3.

\section{Protein expression}

Using the Bac-to-Bac system (Invitrogen), a high titer $\left(10^{9}\right.$ particles per $\left.\mathrm{ml}\right)$ virus encoding the crystallization construct was obtained. Sf9 (Novagen, cat. 71104) cells were infected at a density (23) $\times 10^{6}$ cells per $\mathrm{ml}$ and a multiplicity of infection (MOI) $4-8$, incubated at $28^{\circ} \mathrm{C}, 120 \mathrm{rpm}$ for $50-52 \mathrm{~h}$, harvested by centrifugation at 2,000 $\mathrm{g}$ and stored at $-80{ }^{\circ} \mathrm{C}$ until further use.

\section{Protein purification}

Cells were thawed and lysed by repetitive washes (Dounce homogenization on ice, and centrifugation at $128,600 \mathrm{~g}$ for $30 \mathrm{~min}$ at $\left.4^{\circ} \mathrm{C}\right)$ in hypotonic buffer (10 $\mathrm{mM}$ HEPES pH 7.5, $20 \mathrm{mM} \mathrm{KCl}$, and $10 \mathrm{mM}$ $\left.\mathrm{MgCl}_{2}\right)$ and high osmotic buffer (10 mM HEPES pH 7.5, $20 \mathrm{mM} \mathrm{KCl}, 10 \mathrm{mM} \mathrm{MgCl} 2$, and $\left.1 \mathrm{M} \mathrm{NaCl}\right)$ with an addition of protease inhibitor cocktail [PIC; $500 \mu \mathrm{M}$ 4-(2-aminoethyl)benzenesulfonyl fluoride hydrochloride (Gold Biotechnology), $1 \mu \mathrm{M}$ E-64 (Cayman Chemical), $1 \mu \mathrm{M}$ leupeptin (Cayman Chemical), $150 \mathrm{nM}$ aprotinin (A.G. Scientific)] with the ratio of $50 \mu 1$ per $100 \mathrm{ml}$ of lysis buffer. Membranes were then resuspended in $10 \mathrm{mM}$ HEPES pH 7.5, $20 \mathrm{mM} \mathrm{KCl}, 10 \mathrm{mM} \mathrm{MgCl}_{2}, 2 \mathrm{mg} / \mathrm{ml}$ iodoacetamide, PIC (100 $\mu \mathrm{l}$ per $50 \mathrm{ml}$ of resuspension buffer), and $50 \mu \mathrm{M}$ ONO-5430608 (4-\{6-[2-(1Naphthyl)ethoxy]-1,2,4,5-tetrahydro-3H-3-benzazepin-3-yl butanoic acid; Example $18(18)^{25}$, received as 
a gift from Ono Pharmaceutical) for $30 \mathrm{~min}$ at $4{ }^{\circ} \mathrm{C}$ and then solubilized by addition of $2 \times$ buffer $(50 \mathrm{mM}$ HEPES, $500 \mathrm{mM} \mathrm{NaCl}, 2 \% \mathrm{w} / \mathrm{v}$ n-dodecyl- $\beta$-D-maltopyranoside (DDM; Anatrace), $0.4 \% \mathrm{w} / \mathrm{v}$ cholesteryl hemisuccinate (CHS; Sigma), $10 \% \mathrm{v} / \mathrm{v}$ glycerol) and incubation for $3 \mathrm{~h}$ at $4{ }^{\circ} \mathrm{C}$ with $10 \mathrm{rpm}$ rotation. All further purification steps were performed at $4{ }^{\circ} \mathrm{C}$. The supernatant was clarified by centrifugation $\left(292,055 \mathrm{~g}, 60 \mathrm{~min}, 4^{\circ} \mathrm{C}\right)$ and bound to $2 \mathrm{ml}$ of TALON IMAC resin (Clontech) overnight with $10 \mathrm{rpm}$ rotation in the presence of $20 \mathrm{mM}$ imidazole and $\mathrm{NaCl}$ added up to $800 \mathrm{mM}$. The resin was then washed with ten column volumes (CV) of wash buffer I ( 8 mM ATP, 50 mM HEPES pH 7.5, $10 \mathrm{mM}$ $\mathrm{MgCl}_{2}, 250 \mathrm{mM} \mathrm{NaCl}, 15 \mathrm{mM}$ imidazole, $50 \mu \mathrm{M}$ ONO-5430608, 10 \% v/v glycerol, 0.1/0.02 \%w/v $\mathrm{DDM} / \mathrm{CHS}$ ), then with five CV of wash buffer II (50 mM HEPES pH 7.5, $250 \mathrm{mM} \mathrm{NaCl}, 50 \mathrm{mM}$ imidazole, $50 \mu \mathrm{M}$ ONO-5430608, $10 \% \mathrm{v} / \mathrm{v}$ glycerol, 0.5/0.01\%w/v DDM/CHS), then eluted with (25 mM HEPES pH 7.5, 250 mM NaCl, 400 mM imidazole, $50 \mu \mathrm{M}$ ONO-5430608, 10 \%v/v glycerol, 0.05/0.01\%w/v DDM/CHS) in several fractions. Fractions containing target protein were desalted from imidazole using PD10 desalting column (GE Healthcare) and incubated with $50 \mu \mathrm{M}$ ONO-5430608 and a His-tagged PreScission protease (homemade) overnight with $10 \mathrm{rpm}$ rotation to remove the C-terminal $10 \times$ His tag. Protein was concentrated up to $40-60 \mathrm{mg} / \mathrm{ml}$ using a $100 \mathrm{kDa}$ molecular weight cut-off concentrator (Millipore). The protein purity was checked by SDS-PAGE. Yield and monodispersity were estimated by analytical size exclusion chromatography. Stability and stabilizing effect of the ligand were measured by microscale fluorescent thermal stability assay ${ }^{55}$ (Supplementary Fig. 2).

\section{Thermal Stability Assay}

Microscale fluorescent thermal stability assay ${ }^{55}$ was conducted using a CPM dye (7-Diethylamino-3-(4maleimidophenyl)-4-methylcoumarin, Invitrogen) dissolved in DMF at $10 \mathrm{mM}$. This CPM stock solution was diluted to $1 \mathrm{mM}$ in DMSO and then added to working buffer at $10 \mu \mathrm{M} .1 \mu \mathrm{g}$ of the target protein was added to $50 \mu \mathrm{L}$ of working buffer (25 mM HEPES, $250 \mathrm{mM} \mathrm{NaCl}, 10 \% \mathrm{v} / \mathrm{v}$ glycerol, $0.05 \% \mathrm{w} / \mathrm{v}$ DDM, $0.01 \% \mathrm{w} / \mathrm{v}$ CHS) with CPM, and the melting curve was recorded on a Rotor-Gene Q real-time PCR cycler (Qiagen) using a temperature ramp from 28 to $98^{\circ} \mathrm{C}$ with $2^{\circ} \mathrm{C} / \mathrm{min}$ rate. The fluorescence signal 
was measured in the Blue channel (excitation $365 \mathrm{~nm}$, emission $460 \mathrm{~nm}$ ), and the melting temperature was calculated as the maximum of the fluorescence signal derivative with respect to temperature.

\section{LCP crystallization}

Purified and concentrated $\mathrm{S}_{1} \mathrm{P}_{5}$ was reconstituted in LCP, made of monoolein (Nu-Chek Prep) supplemented with $10 \% \mathrm{w} / \mathrm{w}$ cholesterol (Affymetrix), in 2:3 (v/v) protein:lipid ratio using a syringe lipid mixer ${ }^{27}$. The obtained transparent LCP mixture was dispensed onto 96-wells glass sandwich plates (Marienfeld) in $40 \mathrm{nl}$ drops and covered with $900 \mathrm{nl}$ precipitant using an NT8-LCP robot (Formulatrix) to grow crystals for synchrotron data collection. To prepare crystals for XFEL data collection, the proteinladen LCP mixture was injected into $100 \mu$ Hamilton gas-tight syringes filled with precipitant as previously described ${ }^{27}$. All LCP manipulations were performed at room temperature (RT, 20-23 ${ }^{\circ} \mathrm{C}$ ), while plates and syringes were incubated at $22^{\circ} \mathrm{C}$. Crystals of $\mathrm{S}_{1} \mathrm{P}_{5}$ grew to their full size of $<30 \mu \mathrm{m}$ (in plates) or $<10 \mu \mathrm{m}$ (in syringes) within 3 days in precipitant conditions containing $100-300 \mathrm{mM} \mathrm{KH}_{2} \mathrm{PO}_{4}$ monobasic, 28-32 \%v/v PEG400, and 100 mM HEPES pH 7.0.

\section{Diffraction data collection and structure determination}

$\underline{\text { Synchrotron data collection }}$

Synchrotron data for $\mathrm{S}_{1} \mathrm{P}_{5}-\mathrm{ONO}-5430608$ crystals were collected at the PX1 beamline of the Swiss Light Source of the Paul Scherrer Institute (Villigen, Switzerland). Data were collected at the $1.0 \AA$ wavelength using an Eiger 16M detector with the beam size matching the crystal size, as seen by the initial 2D mesh scan. The data collection was performed using manual X-ray crystal centering: a 2D mesh scan followed by a $1 \mathrm{D}$ mesh scan, selecting the best diffracting spots by visual inspection. The centering was followed by data collection of $90^{\circ}$ with $0.5^{\circ}$ oscillation and a total doze of $30 \mathrm{MGy}$.

Data collection using X-ray free electron laser

XFEL data for $\mathrm{S}_{1} \mathrm{P}_{5}$-ONO-5430608 crystals were collected at the NCI (Nanocrystallography and Coherent Imaging) beamline of the Pohang Accelerator Laboratory X-ray Free Electron Laser (PALXFEL), Pohang, South Korea. The PAL-XFEL was operated in SASE mode at the wavelength of $1.278 \AA$ 
$(9.7 \mathrm{keV})$ and $0.2 \%$ bandwidth, delivering individual X-ray pulses of 25 -fs duration focused into a spot size of $2 \times 3 \mu \mathrm{m}$ using a pair of Kirkpatrick-Baez mirrors. LCP laden with dense suspension of protein microcrystals was injected at room temperature inside a sample chamber filled with helium $\left(23^{\circ} \mathrm{C}, 1 \mathrm{~atm}\right)$ into the beam focus region using an LCP injector ${ }^{56}$ with a $50-\mu \mathrm{m}$-diameter capillary at a flow rate of 0.15 $\mu 1 / \mathrm{min}$. Microcrystals ranged in size from 5 to $10 \mu \mathrm{m}$. Diffraction data were collected at a pulse repetition range of $30 \mathrm{~Hz}$ with a Rayonix MX225-HS detector, operating in a 4x4 binning mode (1440x1440 pixels, $30 \mathrm{fps}$ readout rate). The beam was not attenuated and delivered full intensity $\left(5 \times 10^{11}\right.$ photons per pulse). A total number of 490,000 detector images were collected. Due to a high systematic background, Cheetah ${ }^{57}$ was initially used only to apply dark current calibration, and all images were used for further processing. The overall time of data collection from a sample with a total volume of about $36 \mu$ was approximately 4 hours and yielded 6,918 indexed frames with 7,492 crystal lattices.

\section{$\underline{\text { XFEL data processing with CrystFEL }}$}

During the XFEL data collection, a high systematic background scattering from upstream to the interaction point occurred due to a high intensity X-ray lasing conditions (Supplementary Fig. 4), which prevented from establishing suitable Cheetah hit finding parameters during the beamtime and complicated the overall data processing. All data processing was performed using CrystFEL $0.8 .0^{58}$. Here we describe steps that we took to improve data quality as much as possible starting from the available data with a high background level. For all CrystFEL runs (Supplementary Table 4), peak search was limited with maxres $=340$, min-res $=50$ to search for peaks in the region between the beamstop and the LCP ring, and the frames were limited to a 12,000 subset of all frames, selected with minimum 5 peaks with SNR 2.7. Initially, typical starting peak finding parameters $(S N R=5.0$, threshold $=100)$ in CrystFEL were used for data processing, yielding only 2,036 crystals with indexing=mosflm,dirax,xgandalf (Supplementary Table 4 column A). Initial peak search parameter adjustment, as described in CrystFEL tutorial ${ }^{58}$, led to the value of $\mathrm{SNR}=2.7$ and threshold=30, which yielded 5,275 crystals (Supplementary Table 4 column B). Applying --median-filter $=5$ allowed to further increase the number of crystals to 7,189 , while increasing SNR to 4.0 (Supplementary Table 4 column C). 
Spot integration parameters had the biggest impact on the merged data quality. First, changing spot integration model from rings-nograd model, that assumes flat background around a spot, to rings-grad, that performs $2 \mathrm{D}$-fitting of each spot background profile, decreased overall $\mathrm{R}_{\text {split }}$ from $29.7 \%$ to $19.4 \%$ (Supplementary Table 4 column D) and increased the highest resolution shell CC* from 0.618 to 0.666 Second, increasing local-bg-radius from 3 to 5 , and using int-radius $=3,5,8$ instead of default 4,5,8 further improved data quality with the highest resolution shell CC* equal to 0.716 (Supplementary Table 4, columns E-F). The final merging was performed with partialator, iterations=2, push-res $=5.0$, and model=ggpm $($ Supplementary Table 1$)$.

\section{$\underline{\text { Structure determination and refinement }}$}

The structure was initially solved by molecular replacement using phenix.phaser ${ }^{59}$ with two independent search models of the poly-alanine $\mathrm{S}_{1} \mathrm{P}_{1}$ 7TM domain (PDB ID 3V2Y) and BRIL from the high-resolution $\mathrm{A}_{2 \mathrm{~A}} \mathrm{AR}$ structure (PDB ID 4EIY). Model building was performed by cycling between manual inspection and building with $\operatorname{Coot}^{60}$ v. 0.9.6 using both $2 \mathrm{mFo}-\mathrm{DFc}$ and $\mathrm{mFo}-\mathrm{DFc}$ maps and automatic refinement with phenix.refine ${ }^{61}$ v. 1.19 .2 using automatic torsion-angle NCS restraints and 2 TLS groups. Ligand restraints were generated using the web server GRADE v. 1.2.19 (http://grade.globalphasing.org). The ${\mathrm{S} 1 \mathrm{P}_{5}}_{5}$ structures from two molecules A and B in the asymmetric unit show very high similarity (Ca RMSD $1.0 \AA \AA$ within 7TM; $1.3 \AA$ all-atom RMSD). The main difference includes flexible ECL1 and conformations of several side chains exposed to the lipid bilayer and solvent. The final data collection and refinement statistics are shown in Supplementary Table 1 . The relatively high $\mathrm{R}_{\text {free }}$ of the structure can be explained by high systematic background scattering and the presence of a pseudotranslation-related modulation in the diffraction intensities. The modulation is produced by the NCS operator $(\mathrm{x}, \mathrm{y}, \mathrm{z}) \rightarrow$ $(1 / 8+x,-y,-z)$ as seen in the Patterson maps of both single-crystal and XFEL data: $\sim 0.7$ and 0.3 origin peak high at $(3 / 8,1 / 2,0)$ for single-crystal and serial data, respectively.

\section{AlphaFold predictions}


Prediction runs were executed using AlphaFold ${ }^{50}$ v. 2.1.1+110948 with a non-docker setup (https://github.com/kalininalab/alphafold_non_docker, git commit 7ccdb7) and an updated run_alphafold.sh wrapper with added --random-seed parameter (Supplementary File 1). The use of templates was disabled by setting "max_template_date" to 1900-01-01. 50 AF2-models (ranked_...pdb models) were generated for each of 5 human S1P receptors with protein sequences obtained from UniProt. For each receptor, 10 prediction runs with different seeds (--random-seed"=<run_number $>$ ) were executed; each run generated 5 models. Structures were used as provided by the Alphafold's pipeline with Amber relaxation (see Suppl. Methods 1.8.6 in Ref. 51 for details) without any further modifications.

\section{MD simulations}

Molecular dynamics simulations were conducted for the wild type human $\mathrm{S}_{1} \mathrm{P}_{1}, \mathrm{~S}_{1} \mathrm{P}_{3}$, and $\mathrm{S}_{1} \mathrm{P}_{5}$ receptors based on the X-ray structures $3 \mathrm{~V}_{2} \mathrm{Y}^{19}$ (residues V16-K300), 7C4S $\mathrm{S}^{21}$ (G14-R311), and the structure reported in the present study (S12-C323), respectively. All engineered mutations were reverted back to the WT amino acids, and all missing fragments were filled using MODELLER ${ }^{62}$. Receptors were embedded into lipid bilayers consisting of 1-palmitoyl-2-oleoyl-sn-glycero-3-phosphatidylcholine (POPC) lipids and solvated with TIP3P waters and $\mathrm{Na}^{+} / \mathrm{Cl}^{-}$ions (to guarantee the electroneutrality of the systems and the ionic strength of $0.15 \mathrm{M}$ ) by means of the CHARMM-GUI web-service ${ }^{63}$. The obtained in this way starting models (with 61,666/61,763/56,303 atoms including 119/117/123 POPC molecules in the $\mathrm{S}_{1} \mathrm{P}_{1} / \mathrm{S}_{1} \mathrm{P}_{3} / \mathrm{S}_{1} \mathrm{P}_{5}$ systems, respectively) were subject to standard CHARMM-GUI minimization and equilibration protocol, i.e., the steepest descent minimization (5,000 steps) was followed by a series of short equilibration simulations in the NPT ensemble using Berendsen thermostat and barostat with the restraints on protein and lipids gradually released.

In order to estimate free energy profiles along the rotation of the $\chi_{1}$ torsion angle in the side chain of $\mathrm{Y}^{2.57}$, we employed the metadynamics (metaMD) approach ${ }^{41}$, which is based on addition of biasing repulsive potentials ("hills", typically Gaussians) to the total potential of the system to enhance the sampling of the configurational space along the chosen reaction coordinates. The deposition rate for hills in metaMD 
simulations was $1 \mathrm{ps}^{-1}$; the width and height of deposited hills were equal to $0.1 \mathrm{rad}\left(\sim 5.7^{\circ}\right)$ and 0.5 $\mathrm{kJ} / \mathrm{mol}$, respectively. The metaMD simulations were run for $10 \mathrm{~ns}$ each. To test for convergence of the metaMD simulations, we applied the following $\operatorname{method}^{64}$ : the free energy difference between two regions of the obtained free energy profiles (corresponding to the crystallographic orientations of $\mathrm{Y}^{2.57}$ ) as a function of simulation time were plotted (Supplementary Fig. 12a-c). In case of convergence, this difference should not change with the progress of simulations as the systems diffuse freely along the reaction coordinate.

For the metaMD simulations, Nose-Hoover thermostat and Parrinello-Rahman barostat were used. The temperature and pressure were set to $323.15 \mathrm{~K}$ and 1 bar with temperature and pressure coupling time constants of $1.0 \mathrm{ps}^{-1}$ and $0.5 \mathrm{ps}^{-1}$, respectively. All MD simulations were performed with GROMACS ${ }^{65} \mathrm{v}$. 2020.2 using PLUMED plugin ${ }^{66}$ to enable metaMD. The time step of 2 fs was used for all production simulations. The CHARMM36 force field ${ }^{67}$ was used for the proteins, lipids, and ions.

\section{SAR and molecular docking (with ONO compounds)}

For $\mathrm{S}_{1} \mathrm{P}_{5}$ docking studies, we used chain $\mathrm{B}$ from our $\mathrm{S} \mathrm{P}_{5}-\mathrm{ONO}-5430608$ crystal structure and a metaMD snapshot with the upward conformation of $\mathrm{Y}^{2} 9^{2.57}$. Chain B was selected based on the quality of $2 \mathrm{mFo}-$ DFc maps around the ligand and surrounding residues. Molecular docking was performed using ICM Pro v. 3.9-1b (Molsoft, San Diego). We removed ligands and converted the receptor models into an ICM format using default settings, which includes building missing sidechains, adding hydrogens, energybased Gln/Asn/His conformation optimization, and removal of all water molecules. Same docking box was selected for both models, aligned by their 7TM domains, to encompass both orthosteric and allosteric binding pockets. For each ligand we repeated docking runs 5 times with the effort parameter (ligand sampling depth) set at 16, each time saving 3 best conformations. Ligand structures and their affinities $\left(\mathrm{IC}_{50}\right.$ values from radioligand binding assays) at $\mathrm{S}_{\mathrm{P}} \mathrm{P}_{5}$ receptors were taken from the published patent ${ }^{25}$.

In AlphaFold models analysis, 50 models predicted by the AlphaFold algorithm were compared with both

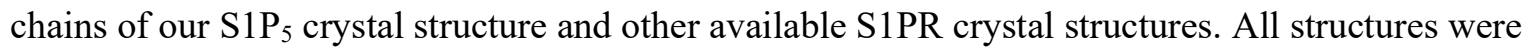


prepared as described above. $\mathrm{S}_{1} \mathrm{P}_{5}$ ligands from $\mathrm{ChEMBL}$ v. 29 were accessed via the web-interface

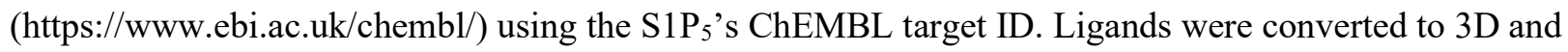
charged at pH 7.0 using Molsoft ICM. For each model, ligand screening was performed three times with docking effort 1. Three ligand benchmarks (Supplementary Fig. 11) were used: 1. "ONO” series: active molecules from Ref. 25 (group A, $1 \mathrm{nM}<\mathrm{IC}_{50}<100 \mathrm{nM}$ ), inactive molecules from Ref. 25 (group C, 1 $\mu \mathrm{M}<\mathrm{IC}_{50}<3 \mu \mathrm{M}$ ) and decoys; 2. "Selective" series: active molecules from Refs. 25,69 (group A or IC $\mathrm{I}_{50}$ $<100 \mathrm{nM}$, correspondingly), inactive molecules from Refs. 25,69 (group C or $\mathrm{IC}_{50}>=1 \mu \mathrm{M}$, correspondingly), and decoys; 3. "Non-selective" series: active molecules from ChEMBL (pChembl >

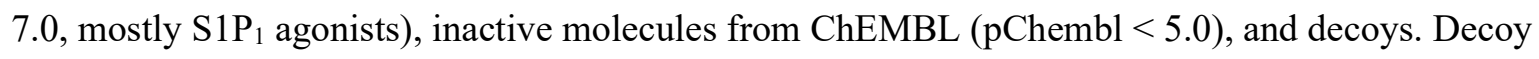
molecules were selected from the Enamine REAL library [https:/enamine.net/compound-collections/realcompounds/real-database], matching the distribution of active molecules by charge and weight. The benchmarks have the following ratios of active:inactive:decoy molecules: 6:5:60 for "ONO", 12:10:120 for "Selective", and 158:39:1207 for "Non-selective", with the imbalance parameter (ratio of the total library size to the number of active molecules in it) of $11.8,11.8$, and 8.8 , respectively. For estimation of the virtual screening quality, metrics enrichment at $10 \%$ and receiver operating characteristic (ROC) area under the ROC curve (AUC) were used, as implemented in RDKIT v. 2021-03-4 (http://www.rdkit.org ${ }^{69}$ ).

\section{Plasmids for functional assays}

The human wild-type S1PR5 gene (UniProt ID Q9H228) with an N-terminal $3 \times$ HA epitope (YPYDVPDYA) tag was cloned into pcDNA3.1+ (Invitrogen) at $\mathrm{KpnI}\left(5^{\prime}\right)$ and $\mathrm{XhoI}\left(3^{\prime}\right)$. Point mutations were introduced by overlapping PCR. All DNA sequences were verified by Sanger sequencing (Evrogen JSC). Sequences of all primers used in this work are listed in Supplementary Table 5.

\section{Cell surface expression determined by ELISA}

Cell surface expression of $\mathrm{S}_{1} \mathrm{P}_{5}$ receptor variants was determined by whole-cell ELISA ${ }^{70}$. Briefly, HEK293T cells were seeded in 24 -well cell culture plates $\left(0.2 \times 10^{6}\right.$ cells in $0.5 \mathrm{ml}$ of medium per well $)$ 
and transfected separately by $3 \mu \mathrm{g}$ of each expression plasmids based on pCDNA3.1(+) vector using common Lipofectamine 3000 protocol. After $12-18 \mathrm{~h}$ incubation in a $\mathrm{CO}_{2}$ incubator at $37{ }^{\circ} \mathrm{C}$ for receptor expression, the cell culture plates were placed on ice, the media was aspirated completely, and the cells were washed once with ice-cold TBS to remove any residual media. Then the cells were fixed using 400 $\mu \mathrm{l}$ of $4 \% \mathrm{w} / \mathrm{v}$ paraformaldehyde (PFA), followed by three $400-500 \mu \mathrm{l}$ washes with TBS. After surface blocking with $2 \% \mathrm{w} / \mathrm{v}$ protease-free BSA (A3059, Sigma) solution in TBS, HRP-conjugated anti-HA antibody high affinity (3F10) (Roche) at a dilution of 1:2000 in TBS $+1 \% \mathrm{w} / \mathrm{v}$ protease-free BSA and TMB ready-to-use substrate (T0565, Sigma) was used for ELISA procedure. The ELISA results were normalized by Janus Green staining. Cells transfected with empty vectors (pCDNA3.1+) were used to determine background.

\section{Functional assays with BRET-based cAMP sensor}

$\mathrm{G}_{\mathrm{i}}$-protein-mediated signaling responses to endogenous agonist S1P and inverse agonist ONO-5430608 were assayed for human WT and mutant S1P5 receptors using Bioluminescence Resonance Energy Transfer (BRET) based EPAC biosensor ${ }^{71}$. Briefly, transfections were carried out by Lipofectamine 3000 according to standard protocol using HEK293T cells seeded in a $100 \mathrm{~mm}$ cell culture plate, receptor cDNA vectors (10 $\mu \mathrm{g}$ each), and EPAC biosensor cDNA vector $(10 \mu \mathrm{g})$ needed for evaluation of cAMP production. Transfected cells were split into 96-well plates at $10^{5}$ cells per well and incubated for $16-18 \mathrm{~h}$. To measure response for S1P, $60 \mu 1$ of PBS was added to each well followed by addition of $10 \mu \mathrm{l}$ of a 50 $\mu \mathrm{M}$ coelenterazine-h, $10 \mu \mathrm{l}$ of $300 \mu \mathrm{M}$ forskolin and $10 \mu \mathrm{l}$ of $100 \mu \mathrm{M}$ 3-isobutyl-1-methylxanthine (IBMX) solutions. After 10-min incubation, either $10 \mu \mathrm{l}$ of vehicle or $10 \mu \mathrm{l}$ of S1P at different concentrations in $0.5 \% \mathrm{w} / \mathrm{v}$ fatty acid-free BSA (10775835001, Roche) solution in PBS was added. To measure response for ONO-5430608, $70 \mu \mathrm{l}$ of PBS was added to each well followed by addition of $10 \mu \mathrm{l}$ of $50 \mu \mathrm{M}$ coelenterazine-h and $10 \mu \mathrm{l}$ of $100 \mu \mathrm{M}$ IBMX solutions. After 10-min incubation, either $10 \mu 1$ of vehicle or $10 \mu \mathrm{l}$ of ONO-5430608 at different concentrations in PBS was added. The plate was then placed into a CLARIOstar reader (BMG LABTECH, Germany) with a BRET filter pair ( $475 \pm 30 \mathrm{~nm}-$ coelenterazine-h and $550 \pm 40 \mathrm{~nm}-$ YFP). The BRET signal was determined by calculating the ratio of the 
light emitted at $550 \mathrm{~nm}$ to the light emitted at $480 \mathrm{~nm}$. The $\mathrm{EC}_{50}$ values were calculated using the threeparameter dose - response curve fit in GraphPad Prism v. 9.3. Three independent experiments were performed in triplicate.

\section{DATA AVAILABILITY}

Data supporting the findings of this manuscript are available from the corresponding authors upon reasonable request. A reporting summary for this article is available as a Supplementary Information file. Coordinates and structure factors have been deposited in the Protein Data Bank (PDB) under the accession code 7YXA. Raw diffraction data have been deposited to CXIDB database (https://www.cxidb.org) under the accession number 196. 


\section{References}

1. An, S., Goetzl, E. J. \& Lee, H. Signaling mechanisms and molecular characteristics of G proteincoupled receptors for lysophosphatidic acid and sphingosine 1-phosphate. J. Cell. Biochem. 30-31, 147-57 (1998).

2. Maceyka, M., Harikumar, K. B., Milstien, S. \& Spiegel, S. Sphingosine-1-phosphate signaling and its role in disease. Trends Cell Biol. 22, 50-60 (2012).

3. Thuy, A. v., Reimann, C. M., Hemdan, N. Y. A. \& Gräler, M. H. Sphingosine 1-phosphate in blood: Function, metabolism, and fate. Cell. Physiol. Biochem. 34, 158-71 (2014).

4. LaMontagne, K. et al. Antagonism of sphingosine-1-phosphate receptors by FTY720 inhibits angiogenesis and tumor vascularization. Cancer Res. 66, 221-31 (2006).

5. Hla, T. Signaling and biological actions of sphingosine 1-phosphate. Pharmacol. Res. 47, 401-7 (2003).

6. Inoue, A. et al. Illuminating G-Protein-Coupling Selectivity of GPCRs. Cell. 177, 1933-1947.e25 (2019).

7. Takuwa, N., Okamoto, Y., Yoshioka, K. \& Takuwa, Y. Sphingosine-1-phosphate signaling and cardiac fibrosis. Inflamm. Regen. 33, 96-108 (2013).

8. Im, D. S. et al. Characterization of a novel sphingosine 1-phosphate receptor, Edg-8. J. Biol. Chem. 275, 14281-6 (2000).

9. Liu, H. et al. Sphingosine-1-phosphate modulates PAR1-mediated human platelet activation in a concentration-dependent biphasic manner. Sci. Rep. 11, 15308 https://doi.org/10.1038/s41598-02194052-4 (2021).

10. Schmidt, K. G. et al. Sphingosine-1-phosphate receptor 5 modulates early-stage processes during fibrogenesis in a mouse model of systemic sclerosis: A pilot study. Front. Immunol. 8, 1242 https://doi.org/10.3389/fimmu.2017.01242 (2017).

11. Hobson, A. D. et al. Discovery of A-971432, an orally bioavailable selective sphingosine-1phosphate receptor 5 (S1P5) agonist for the potential treatment of neurodegenerative disorders. $J$. Med. Chem. 58, 9154-9170 (2015).

12. Shah, M. V. et al. Molecular profiling of LGL leukemia reveals role of sphingolipid signaling in survival of cytotoxic lymphocytes. Blood. 112, 770-81 (2008).

13. Bargiela, N. F., García, C. M., Arufe, V. G., Hermida, J. R. V. \& Herranz, I. M. Fingolimod in multiple sclerosis: Profile of use in habitual practice. Eur. J. Hosp. Pharm. 27, 346-349 (2020).

14. Synnott, P. G., Bloudek, L. M., Sharaf, R., Carlson, J. J. \& Pearson, S. D. The Effectiveness and Value of Siponimod for Secondary Progressive Multiple Sclerosis. J. Manag. Care Spec. Pharm. 26, 236-239 (2020).

15. Lamb, Y. N. Ozanimod: First Approval. Drugs. 80, 841-848 (2020).

16. Kurata, H. et al. Discovery of a 1-Methyl-3,4-dihydronaphthalene-Based Sphingosine-1-Phosphate (S1P) Receptor Agonist Ceralifimod (ONO-4641). A S1P1 and S1P5 Selective Agonist for the Treatment of Autoimmune Diseases. J. Med. Chem. 60, 9508-9530 (2017).

17. Pérez-Jeldres, T. et al. Targeting cytokine signaling and lymphocyte traffic via small molecules in inflammatory bowel disease: JAK inhibitors and S1PR agonists. Front. Pharmacol. 10, 212 https://doi.org/10.3389/fphar.2019.00212 (2019).

18. Sanna, M. G. et al. Enhancement of capillary leakage and restoration of lymphocyte egress by a chiral S1P1 antagonist in vivo. Nat. Chem. Biol. 2, 434-41 (2006).

19. Hanson, M. A. et al. Crystal structure of a lipid G protein-coupled receptor. Science. 335, 851-5 (2012).

20. Scott, F. L. et al. Ozanimod (RPC1063) is a potent sphingosine-1-phosphate receptor-1 (S1P1) and receptor-5 (S1P5) agonist with autoimmune disease-modifying activity. Br. J. Pharmacol. 173, 1778-92 (2016).

21. Maeda, S. et al. Endogenous agonist-bound S1PR3 structure reveals determinants of G proteinsubtype bias. Sci. $A d v$. 7, eabf5325 https://doi.org/10.1126/sciadv.abf5325 (2021).

22. Zhao, C. et al. Structural insights into sphingosine-1-phosphate recognition and ligand selectivity of S1PR3-Gi signaling complexes. Cell Res. 32, 218-221 (2022).

23. Yuan, Y. et al. Structures of signaling complexes of lipid receptors S1PR1 and S1PR5 reveal mechanisms of activation and drug recognition. Cell Res. 31, 1263-1274 (2021).

24. Xu, Z. et al. Structural basis of sphingosine-1-phosphate receptor 1 activation and biased agonism. Nat. Chem. Biol. https://doi.org/10.1038/s41589-021-00930-3 (2021). 
25. Watanabe, T., Kusumi, K. \& Yuichil Inagaki, I. (2017). Tetrahydronaphthalene Derivative. United States patent No. US 2019/0031605A1 U.S. Patent and Trademark Office https://patentscope.wipo.int/search/en/detail.jsf?docId=US236791513

26. Chun, E. et al. Fusion partner toolchest for the stabilization and crystallization of $\mathrm{G}$ protein-coupled receptors. Structure. 20, 967-76 (2012).

27. Caffrey, M. \& Cherezov, V. Crystallizing membrane proteins using lipidic mesophases. Nat. Protoc. 4, 706-731 (2009)

28. Chrencik, J. E. et al. Crystal Structure of Antagonist Bound Human Lysophosphatidic Acid Receptor 1. Cell. 161, 1633-43 (2015).

29. Ballesteros, J. A. \& Weinstein, H. Integrated methods for the construction of three-dimensional models and computational probing of structure-function relations in $\mathrm{G}$ protein-coupled receptors. Methods Neurosci. 25, 366-428 (1995).

30. Zhou, Q. et al. Common activation mechanism of class a GPCRs. eLife 8, e50279 (2019).

31. Hilger, D. The role of structural dynamics in GPCR-mediated signaling. FEBS J. 288, 2461-2489 (2021).

32. Schoepp, N. G. et al. Crystal structures of agonist-bound human cannabinoid receptor CB1. Nature. 55, 468-471 (2017).

33. Shao, Z. et al. Structure of an allosteric modulator bound to the CB1 cannabinoid receptor. Nat. Chem. Biol. 15, 1199-1205 (2019).

34. Wingler, L. M. et al. Angiotensin and biased analogs induce structurally distinct active conformations within a GPCR. Science. 367, 888-892 (2020).

35. Israeli, H. et al. Structure reveals the activation mechanism of the MC4 receptor to initiate satiation signaling. Science. 372, 808-814 (2021).

36. Katritch, V. et al. Allosteric sodium in class A GPCR signaling. Trends Biochem. Sci. 39, 233-44 (2014).

37. Zarzycka, B., Zaidi, S. A., Roth, B. L. \& Katritch, V. Harnessing ion-binding sites for GPCR pharmacology. Pharmacol. Rev. 71, 571-595 (2019).

38. Katritch, V., Cherezov, V. \& Stevens, R. C. Structure-function of the G protein-coupled receptor superfamily. Annu. Rev. Pharmacol. Toxicol. 53, 531-56 (2013).

39. Nygaard, R., Frimurer, T. M., Holst, B., Rosenkilde, M. M. \& Schwartz, T. W. Ligand binding and micro-switches in 7TM receptor structures. Trends Pharmacol. Sci. 30, 249-59 (2009).

40. Fujiwara, Y. et al. Identification of the hydrophobic ligand binding pocket of the S1P 1 receptor. $J$. Biol. Chem. 282, 2374-85 (2007).

41. Laio, A. \& Parrinello, M. Escaping free-energy minima. Proc. Natl. Acad. Sci. U S A. 99, $12562-$ 12566 (2002).

42. Niedernberg, A., Blaukat, A., Schöneberg, T. \& Kostenis, E. Regulated and constitutive activation of specific signalling pathways by the human S1P 5 receptor. Br. J. Pharmacol. 138, 481-493 (2003).

43. Karczewski, K. J. et al. The mutational constraint spectrum quantified from variation in 141,456 humans. Nature. 581, 434-443 (2020).

44. Tate, J. G. et al. COSMIC: The Catalogue Of Somatic Mutations In Cancer. Nucleic Acids Res. 47, D941-D947 (2019).

45. Hornik, J. Analysis of naturally occurring genetic variants in the sphingosine-1-phosphate receptor family. PhD Thesis. The Pennsylvania State University, University Park (2019).

46. Arakawa, M. et al. Structural and functional roles of small group-conserved amino acids present on helix-H7 in the $\beta 2$-adrenergic receptor. Biochim. Biophys. Acta-Biomembranes 1808, 1170-8 (2011).

47. Steen, A. et al. Biased and constitutive signaling in the CC-chemokine receptor CCR5 by manipulating the interface between transmembrane helices 6 and 7. J. Biol. Chem. 288, 12511-21. (2013).

48. Wang, J. et al. The structural study of mutation-induced inactivation of human muscarinic receptor M4. IUCrJ 7, 294-305 (2020).

49. Bertalan, É., Lešnik, S., Bren, U. \& Bondar, A. N. Protein-water hydrogen-bond networks of G protein-coupled receptors: Graph-based analyses of static structures and molecular dynamics. $J$. Struct. Biol. 212, 107634 https://doi.org/10.1016/j.jsb.2020.107634 (2020).

50. Jumper, J. et al. Highly accurate protein structure prediction with AlphaFold. Nature. 596, 583-589 (2021). 
51. Akdel, M. et al. A structural biology community assessment of AlphaFold 2 applications. Preprint at https://www.biorxiv.org/content/10.1101/2021.09.26.461876v1 (2021).

52. Yuan, S., Wu, R., Latek, D., Trzaskowski, B. \& Filipek, S. Lipid Receptor S1P1 Activation Scheme Concluded from Microsecond All-Atom Molecular Dynamics Simulations. PLoS Comput. Biol. 9, e1003261 https://doi.org/10.1371/journal.pcbi.1003261 (2013).

53. Heo, L. \& Feig, M. Multi-State Modeling of G-protein Coupled Receptors at Experimental Accuracy. Preprint at https://www.biorxiv.org/content/10.1101/2021.11.26.470086v1 (2021).

54. Tong, A. B. et al. Could AlphaFold revolutionize chemical therapeutics? Nat. Struct. Mol. Biol. 28, 771-772 (2021).

55. Alexandrov, A. I., Mileni, M., Chien, E. Y. T., Hanson, M. A. \& Stevens, R. C. Microscale fluorescent thermal stability assay for membrane proteins. Structure. 16, 351-9 (2008).

56. Weierstall, U. et al. Lipidic cubic phase injector facilitates membrane protein serial femtosecond crystallography. Nat. Commun. 5, 3309 https://doi.org/10.1038/ncomms4309 (2014).

57. Barty, A. et al. Cheetah: Software for high-throughput reduction and analysis of serial femtosecond X-ray diffraction data. J. Appl. Crystallogr. 47, 1118-1131 (2014).

58. White, T. A. et al. CrystFEL: A software suite for snapshot serial crystallography. J. Appl. Crystallogr. 45, 335-341 (2012).

59. McCoy, A. J. et al. Phaser crystallographic software. J. Appl. Crystallogr. 40, 658-674 (2007).

60. Emsley, P., Lohkamp, B., Scott, W. G. \& Cowtan, K. Features and development of Coot. Acta Crystallogr. D Biol. Crystallogr. 66, 486-501 (2010).

61. Afonine, P. v. et al. Towards automated crystallographic structure refinement with phenix.refine. Acta Crystallogr. D Biol. Crystallogr. 68, 352-67 (2012).

62. Webb, B. \& Sali, A. Comparative protein structure modeling using MODELLER. Curr. Protoc. Bioinformatics. 54, 5.6.1-5.6.37 https://doi.org/10.1002/cpbi.3 (2016).

63. Jo, S., Kim, T., Iyer, V. G. \& Im, W. CHARMM-GUI: A web-based graphical user interface for CHARMM. J. Comput. Chem. 29, 1859-65 (2008).

64. Pfaendtner, J., Branduardi, D., Parrinello, M., Pollard, T. D. \& Voth, G. A. Nucleotide-dependent conformational states of actin. Proc. Natl. Acad. Sci. U S A. 106, 12723-12728 (2009).

65. Abraham, M. J. et al. Gromacs: High performance molecular simulations through multi-level parallelism from laptops to supercomputers. SoftwareX 1-2, 19-25 (2015).

66. Tribello, G. A., Bonomi, M., Branduardi, D., Camilloni, C. \& Bussi, G. PLUMED 2: New feathers for an old bird. Comput. Phys. Comm. 185, 604-613 (2014).

67. Huang, J. et al. CHARMM36m: An improved force field for folded and intrinsically disordered proteins. Nat. Methods. 14, 71-73 (2016).

68. Ma, B. et al. Novel Potent Selective Orally Active S1P5 Receptor Antagonists. ACS Med. Chem. Lett. 12, 351-355 (2021).

69. Landrum, G. (2014) RDKit: open-source cheminformatics. https://doi.org/10.5281/ZENODO.10398

70. Pandey, S., Roy, D. \& Shukla, A. K. Measuring surface expression and endocytosis of GPCRs using whole-cell ELISA. Methods Cell Biol. 149, 131-140 (2019).

71. Salahpour, A. et al. BRET biosensors to study GPCR biology, pharmacology, and signal transduction. Front. Endocrinol. 3, 105 https://doi.org/10.3389/fendo.2012.00105 (2012). 


\section{Acknowledgements}

We thank S. Ustinova, A. Podzorov, A. Awawdeh, P. Utrobin, and Yu. Semenov for technical assistance, T. Maruyama for providing ONO-5430608. We acknowledge the Paul Scherrer Institut, Villigen, Switzerland for provision of synchrotron radiation beamtime at beamline PX1 of the SLS and would like to thank Dr. V. Olieric for assistance. The authors are grateful to the staff of the accelerator and beamline departments at PAL-XFEL for their technical support. The XFEL experiments were performed at the NCI PAL-XFEL experimental station under the proposal No. 2019-2nd-NCI-012. Protein production and crystallization was supported by the Russian Science Foundation project no. 19-14-00261. Synchrotron data collection and treatment were supported by the Russian Ministry of Science and Higher Education grant No. 075-152021-1354. SFX data collection strategy was developed with the support from the Russian Foundation for Basic Research (RFBR, project № 18-02-40020). Functional assays were implemented with the support of the Ministry of Science and Higher Education of the Russian Federation (agreement \# 075-00337-20-03, project FSMG-2020-0003). J.P. and Y.C. were supported by the National Research Foundation of Korea (grant No. NRF-2017M3A9F6029736). The authors are grateful to the Global Science Experimental Data Hub Center (GSDC) for data computing and the Korea Research Environment Open NETwork (KREONET) for network service provided by the Korea Institute of Science and Technology Information (KISTI) and to the Data Processing Center of Moscow Institute of Physics and Technology for highperformance data computing infrastructure and technical support. V.C. acknowledges that the University of Southern California is his primary affiliation.

\section{Author contributions}

E.L. and A.Gu. optimized the constructs, developed the expression and purification procedure, expressed and purified the protein, screened the ligands, and crystallized the protein-ligand complexes.

E.M., D.V., A.M. and V.C. collected X-ray diffraction data at PAL XFEL.

J.P. set up the XFEL experiment, beamline, controls, and data acquisition; operated the beamline. H.H., U.W. helped develop and operate the LCP injector. W.L. helped with the XFEL sample preparation. S.P. contributed to the experimental system installation at the beamline. G.P. 
contributed to DAQ and data handling. H.J.H. contributed to the Rayonix detector installation and operation.

A.Gu., E.M., E.L., K.K and A.M. collected synchrotron data at SLS.

A.Ge. performed and analyzed cell signaling and cell surface expression assays.

E.M., D.V. and V.B. processed diffraction data.

G.B. helped with X-ray diffraction data interpretation and analysis.

E.M., V.B., V.C. performed structure determination and refinement.

V.B., V.C., E.L., A.Gu., E.M., A.M., A.L. performed project data analysis/interpretation.

M.E., A.L., M.S. helped with construct optimization, protein expression and purification.

P.P. advised on the protein construct design.

E.M., M.K. performed molecular docking.

P.O., E.M. performed MD simulations.

E.M., I.G., V.B. performed AlphaFold simulations and their data analysis.

A.L., I.O., P.Kh., A.R., Y.C. helped with experimental work and project organization.

E.L., E.M., A.Gu., P.O., A.M., V.B., V.C., wrote the manuscript with the help from other authors.

V.C, A.M., V.B. and V.G. initiated the project.

A.M. and V.B. organized the project implementation, were responsible for the overall project management, and co-supervised the research.

V.C. supervised the overall project.

\section{Competing interests}

Authors declare no competing interests. 


\section{FIGURES AND TABLES}

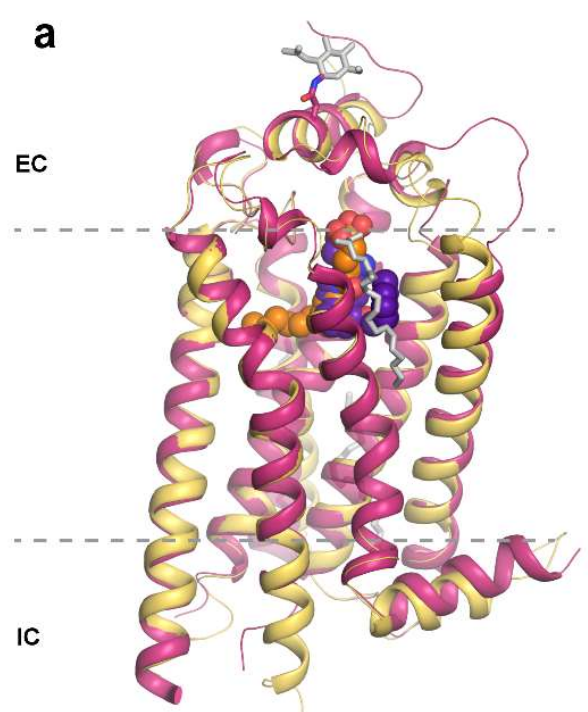

C
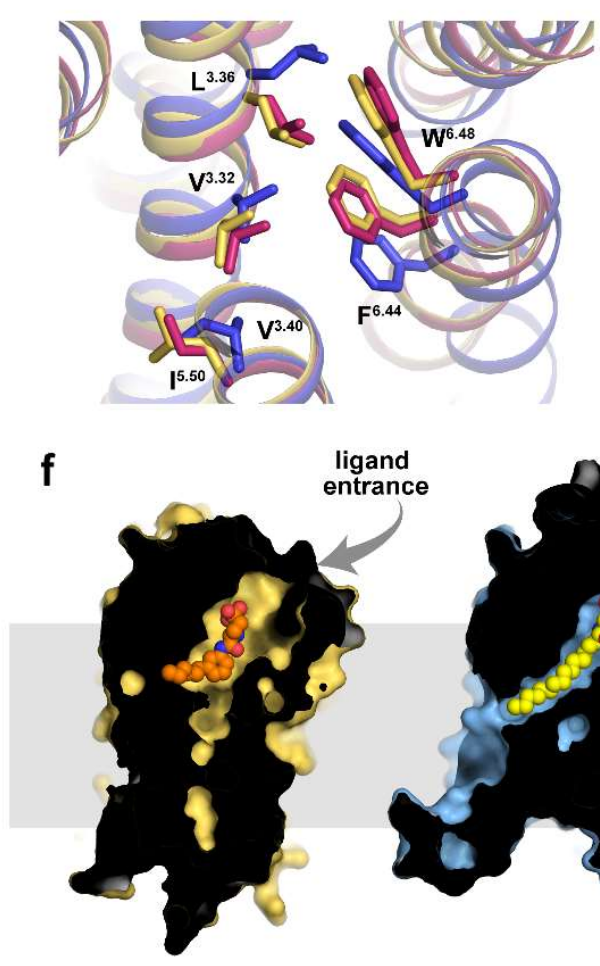

S1P 1 inactive
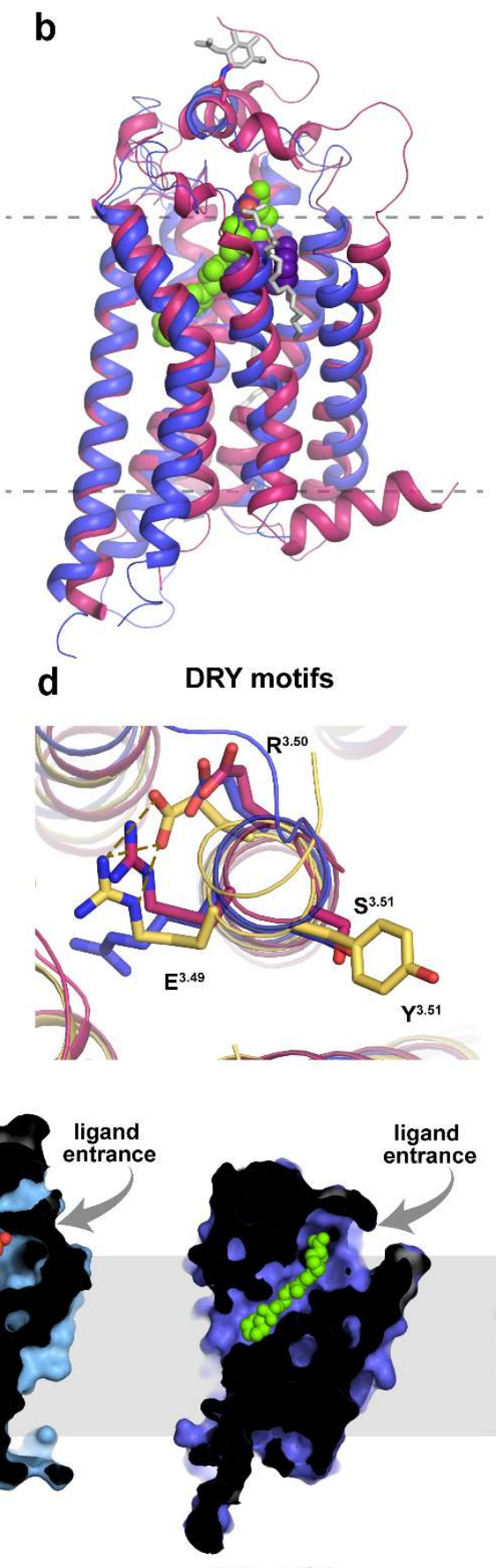

S1P $\mathrm{P}_{5}$ active

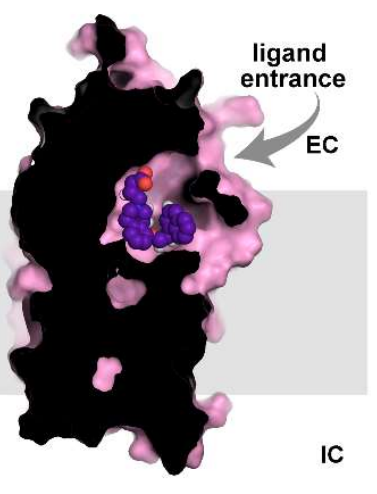

S1 $P_{5}$ inactive

Fig. 1 Structure of S1P5 and its comparison with structures of other S1PRs: overview and conservative motifs. a Superposition of the obtained in this work inactive $\mathrm{S}_{1} \mathrm{P}_{5}$ structure (pink cartoon) in complex with ONO-5430608 (purple spheres) with the inactive $\mathrm{S}_{1} \mathrm{P}_{1}$ (yellow cartoon)-ML056 (orange spheres) complex (PDB ID 3V2Y). b Superposition of the inactive 


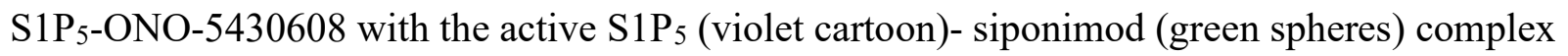

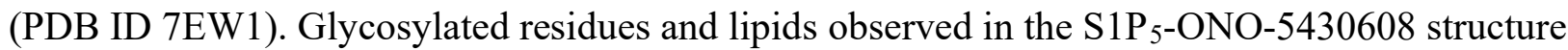
are shown as gray sticks. $\mathbf{c}$ Superposition of the dual toggle switch and P-I-F motif for $\mathrm{S}_{1} \mathrm{P}_{5-}$

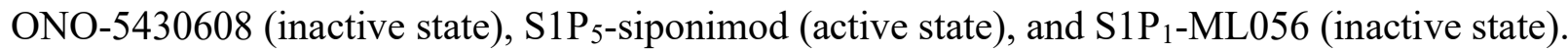
d Superposition of the DRY functional motif for the same three receptor-ligand pairs as in (c). e Sequence conservation of the transmission switches and DRY motif in the SP1R family. Adenosine $\mathrm{A}_{2 \mathrm{~A}}$ receptor is included as a representative receptor of class A GPCR. f Sliced surface representation of known structures from the S1PR family with corresponding ligands: S1P1-ML056 (PDB ID 3V2Y), S1P 3 -S1P (PDB ID 7C4S), S1P 5 -siponimod (PDB ID 7EW1), and $\mathrm{S}_{\mathrm{P}} \mathrm{F}_{5} \mathrm{ONO} 5430608$ (this work, PDB ID 7YXA). 


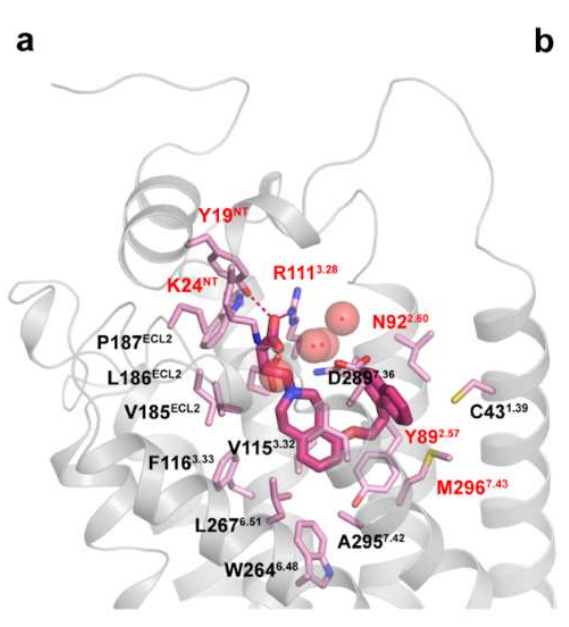

b

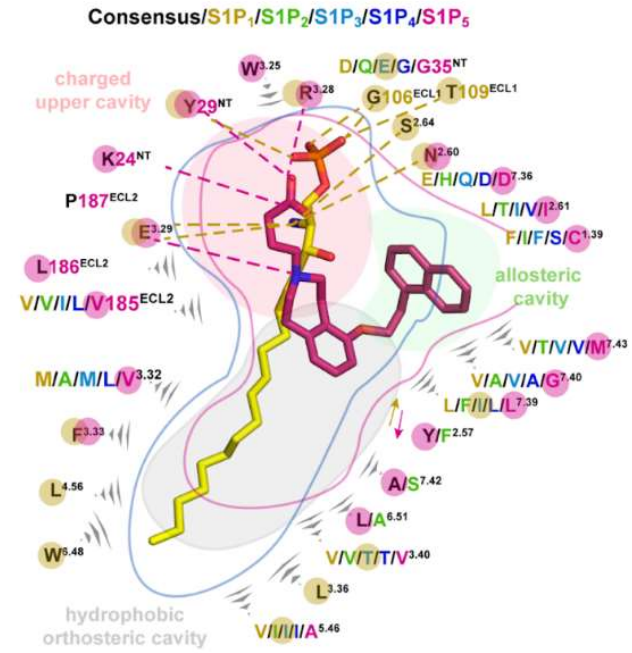

C

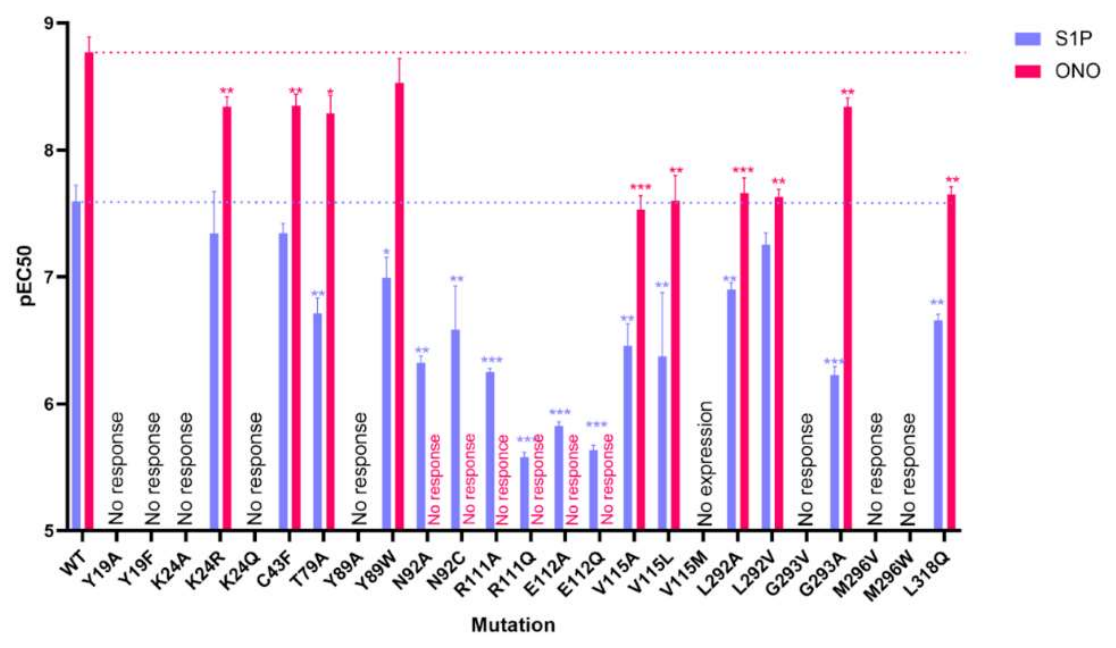

Fig. 2 Structural and functional comparison between ONO-5430608 and S1P binding.

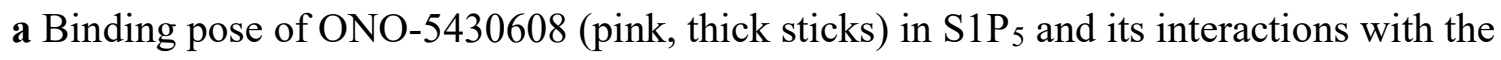
receptor residues (light pink, thin sticks). Polar interactions are shown as dashed lines. Residues which had mutations disrupting response to ONO-5340608 are labeled in red. b Schematic diagram of the ligand binding pocket and interactions between ONO-5430608 and $\mathrm{S}_{1} \mathrm{P}_{5}$ (this work, PDB ID 7YXA) compared to interactions between S1P and S1P 3 (PDB ID 7C4S). Residues are color coded according to different $\mathrm{S} 1 \mathrm{P}$ receptor subtypes $\left(\mathrm{S}_{1} \mathrm{P}_{1}\right.$ - yellow, $\mathrm{S}_{\mathrm{P}} \mathrm{P}_{2}$ -

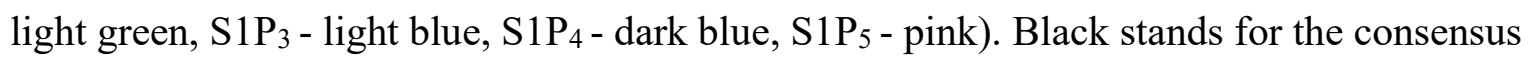
residue shared by all receptors. Residues interacting with ONO-5430608 are highlighted with pink circles, residues interacting with S1P are highlighted with yellow circles. Polar interactions are shown as dashed lines. c Potencies ( $\mathrm{pEC}_{50}$ ) of S1P (purple, agonism) and ONO-5430608 (pink, antagonism) at WT and mutants of $\mathrm{S} \mathrm{P}_{5}$ in $\mathrm{G}_{\mathrm{i}}$ protein-mediated signaling assays. Data are 
bioRxiv preprint doi: https://doi.org/10.1101/2022.02.25.480536; this version posted February 26, 2022. The copyright holder for this preprint (which was not certified by peer review) is the author/funder, who has granted bioRxiv a license to display the preprint in perpetuity. It is made available under aCC-BY 4.0 International license.

shown as mean \pm s.d. of 3 independent experiments conducted in triplicates. $* 10^{-2} \leq p<510^{-2}$, $* * 10^{-3} \leq p<10^{-2}, * * * p<10^{-3}$. Dose-response curves are shown in Supplementary Fig. 6. 
a

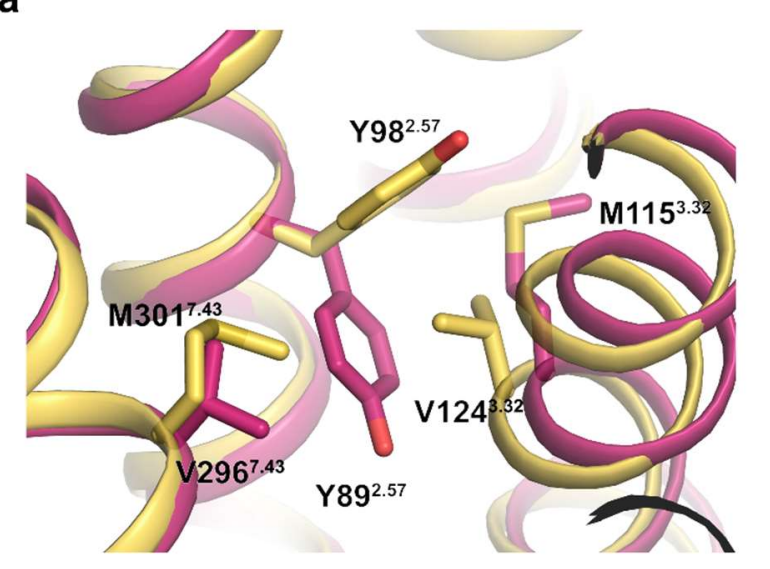

b

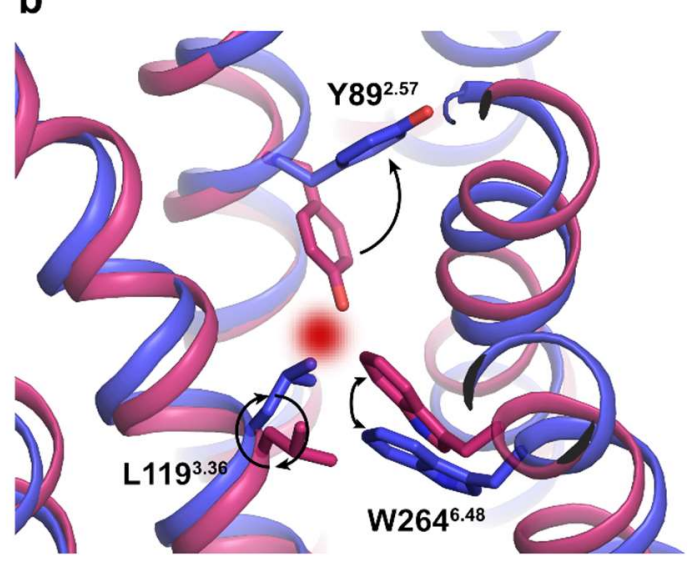

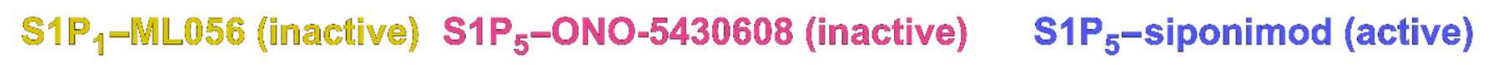
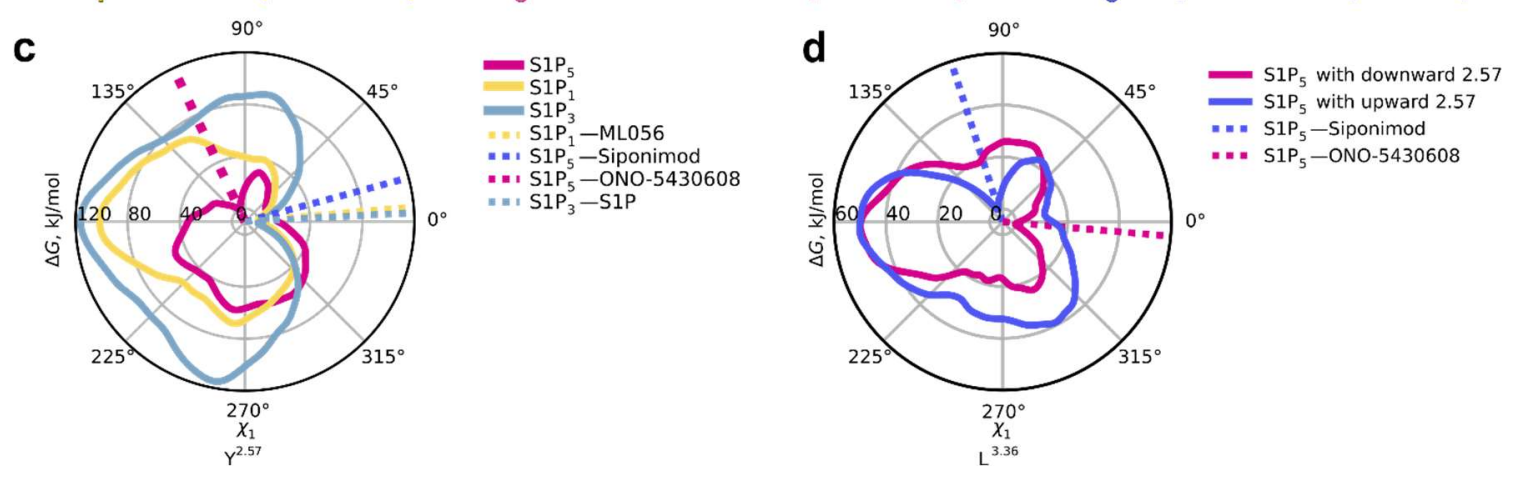

Fig. 3 Conformational flexibility of $\mathrm{Y}^{2.57}$ and its effect on inverse agonism in S1P receptors. a Two distinct upward and downward conformations of $\mathrm{Y}^{2.57}$ as observed in crystal structures of S1P1-ML056 (PDB ID 3V2Y) and S1P 5 -ONO-5430608 (this work, PDB ID 7YXA), respectively. $\mathbf{b}$ The downward orientation of $\mathrm{Y}^{2.57}$ is incompatible with the active state of the dual toggle switch $\mathrm{L}^{3.36}-\mathrm{W}^{6.48}$ because of a steric clash. $\mathrm{S}^{\mathrm{P}} \mathrm{P}_{5}$-ONO-5430608 (this work, PDB ID 7YXA, inactive state) is shown in pink and $\mathrm{S}_{1} \mathrm{P}_{5}$-siponimod (PDB ID 7EW1, active state) is shown in purple. $\mathbf{c}$ Free energy profiles of the $\mathrm{Y}^{2.57}$ side chain torsion angle $\chi_{1}$ as calculated by metaMD for $\mathrm{S}_{1} \mathrm{P}_{1}, \mathrm{~S}_{1} \mathrm{P}_{3}$ and $\mathrm{S}_{1} \mathrm{P}_{5}$. Dotted lines correspond to $\mathrm{Y}^{2.57}$ conformations in corresponding experimental structures. $\mathbf{d}$ Free energy profiles of the $\mathrm{L}^{3.36}$ side chain torsion angle in $\mathrm{S}_{1} \mathrm{P}_{5}$ with two alternative orientations of $\mathrm{Y}^{2.57}$ as calculated by metaMD. Dotted lines correspond to $\mathrm{L}^{3.36}$ conformations in corresponding experimental structures. 